\title{
Aero-Structural Coupling Strategy for a Morphing Blade Cascade Study
}

\author{
Giada Abate *广 \\ Researcher \\ German Aerospace Center (DLR) \\ Institute of Composite Structures \\ and Adaptive Systems \\ Lilienthalpl. 7, 38108 Braunschweig, \\ Germany \\ Email: giada.abate@dlr.de
}

\author{
Johannes Riemenschneider \\ Head of Department \\ German Aerospace Center (DLR) \\ Institute of Composite Structures \\ and Adaptive Systems \\ Lilienthalpl. 7, 38108 Braunschweig, \\ Germany
}

\author{
Alexander Hergt \\ Researcher \\ German Aerospace Center (DLR) \\ Institute of Propulsion Technology \\ Porz-Wahnheide, Linder Höhe, 51147 Köln, Germany
}

\begin{abstract}
The coupling of aerodynamics and structural mechanics is an important step in the design process of aeronautical devices with morphing parts. In this paper, a 2D-3D coupling approach is developed to study a morphing blade cascade. Two shape memory alloy actuators are placed on the upper and lower sides of the blade to make possible the change in shape of the leading-edge. In the present work, a preliminary design study is conducted by considering a two-dimensional computational fluid dynamics (CFD) analysis of an airfoil cascade coupled with a three-dimensional structural analysis of the whole $3 D$ blade. $A$ methodology is developed to match $2 D$ and $3 D$ meshes such that the aerodynamic loads can be easily transferred to the structural analysis. From there, the deformed blade geometry due to both aerodynamic loads and actuator work can be transferred back to the CFD solver, and the iterative aero-structural coupling loop can be repeated until convergence. The aero-structural coupling strategy developed in this work is also applied to a blade cascade study aiming to improve its performance by morphing the leading-edge of the blade. The results of this application show that by morphing the leading-edge blade of only few millimeters (less than $2 \mathrm{~mm}$ ), it is possible to achieve a relevant performance improvement
\end{abstract}

*Address all correspondence for other issues to this author.

${ }^{\dagger}$ Address all correspondence related to ASME style format and figures to this author. 
in terms of total pressure loss coefficient decrease of about 53\% considering off-design conditions.

\section{INTRODUCTION}

Shape adaptation or morphing of surfaces is a concept deriving from the observation of nature [1]. For examples, birds and insects are able to achieve a wide range of flight conditions by changing their wing shape. In a similar way, morphing wings and aeronautical engine blades have been studied with the final goal of improving the aircraft performance in off-design conditions. Morphing has the potential to bring numerous advantages in comparison to a rigid conventional solution. For example, aeronautical engines work with fixed blade geometries, and since the engine operates at design and off-design conditions, the design process always results as a compromise between these two operations. The morphing concept in this case permits to design adaptive engine blade geometries able to modify their shape according to the aerodynamic requirements at the given operating condition, thereby leading to an increase of aerodynamic efficiency, which means a potential reduction in fuel consumption. The application of the morphing concept to engine blades has been studied in several works. For example in [2], the inlet guide vanes of a compressor are morphed such that they actively adapt their geometries to adjust the desired flow conditions. For this purpose, smart materials [3,4] are used due to their capability to introduce stresses and strains into a structure when subjected to electric, thermal or magnetic loads. Smart materials have great potential for such application because they can be attached to the engine blades or even integrated inside the structure for a more compact and lightweight design. Several studies on smart materials and morphing devices demonstrate their positive contribution to the engine performance: piezoelectric layers are able to reduce vibrations [5, 6, 2], active trailing-edge blowing control [7, 2] can limit the unsteady stator-rotor interaction, a blade tip clearance control [2] is applied to reduce losses, and piezoelectric actuators [8,9] placed on the upper and lower sides of the blade when activated can change the camber angle and therefore the stagger angle of the blade. Smart materials like shape memory alloys are also successfully applied to aeronautical structures with relevant improvement in flight performance $[10,11,12,13]$. The main challenge in designing morphing blades is to satisfy the contrasting requirements imposed by two disciplines: structural mechanics and aerodynamics. On one hand, the structure has to be stiff enough to withstand the aerodynamic loads while maintaining the prescribed aerodynamic properties; on the other hand, it has to be compliant enough to allow shape changes. The result is a compromise between the two requirements. Moreover, the two above mentioned disciplines (aerodynamics and structural mechanics) are highly coupled in the design process of morphing aeronautical components, therefore, it is very important to introduce in the process a methodology able to link these two disciplines together. For this purpose, several fluid-structure interaction (FSI) methods have been developed to achieve a more complete and reliable design process of morphing devices.

According to [14], the classical approach to solve aeroelastic problems relies on iterative architectures with coupled aerodynamic and structural models. Indeed, the aerodynamic loads need to be transferred to the structural solver which requires a complete description of the force field to provide an accurate geometry deformation. On the other hand, the geometry shape affects the aerodynamics, and therefore the computed pressure field by the CFD solver. The procedure continues until convergence. The aeroelastic coupling developed in [14] is done through a dedicated interface procedure integrated in an optimization framework for a morphing wing. The virtual work principle is implemented in the structural analysis and the resulting displacements are used to 
update the boundary conditions of the aerodynamic model. Once the convergence is achieved, the final aeroelastic shape is then computed.

Another example of aerodynamics-structures coupling is presented in [15], where a CFD code is linked to a rotorcraft computational structural dynamics (CSD) code to calculate helicopter rotor airloads across a range of flight conditions. According to [15], the coupling between CFD and rotorcraft comprehensive codes can be accomplished in two ways. In the loose (weak) coupling methodology, information between CFD and CSD is transferred on a per-revolution basis; in the tight (strong) coupling approach, the CFD and CSD codes are coupled at every time step and integrated simultaneously.

Another fluid-structure coupling process is conducted in [16] with a development of an efficient and robust grid deformation technique, an accurate data interpolation to transfer information from CFD to structural mesh grids and vice versa, as well as the implementation of suitable interfaces between CFD and structural analysis solvers. The coupling procedure is designed for steady and unsteady fluid-structure interactions. The authors in [16] demonstrate the significant impact that the FSI has on the aerodynamic analysis and design of transport and combat aircraft.

Another example of FSI is reported in [17], where an aerodynamic shape optimization code is coupled with a structural morphing model and they are used to obtain a set of optimal wing shapes for minimum drag at different flight speeds. The aerodynamic shape optimization code is suitable for preliminary wing optimization tasks; from a given flight condition and aircraft weight, the aerodynamic optimization tool finds the optimal wing shape which is passed with the aerodynamic loads to the structural model. At this point the process becomes iterative: the structural control points of the morphing ribs are made coincident to the aerodynamic control points, and the aerodynamic loads are distributed to the skin of the structural model; the structural analysis is carried out and the deformation of the skin is obtained. In the next iteration, the new wing shape is passed to an aerodynamic solver and new loads are computed. The process stops when the convergence is achieved.

A multidisciplinary static aeroelastic analysis tool for morphing airfoils is developed in [18]. This tool is able to identify a set of parameters describing the airfoil geometry, its internal structure and its actuators, that allow the profile to be deformed and reach the specified aerodynamic performance while maximizing an aerodynamic and structural performance index [18]. The key aspects of that approach are to optimize concurrently the aerodynamic and structural parameters describing the airfoil and the ability to consider the static aeroelastic coupling in the analysis of its behavior. In the coupling process, the pressure acting on the airfoil surface is transformed into forces acting on the nodes delimiting each structural panel; the aerodynamic model shape is updated by moving the aerodynamic nodes according to the displacements of the neighboring structural nodes resulting from the finite element analysis. In this way, the structural and the aerodynamic models are not forced to be based on the same nodes, but they can have different levels of refinement.

Another work by Molinari [19] proposes a design methodology for 3D morphing wings based on the concurrent optimization of structural and aerodynamic design variables. In the aeroelastic coupling, the pressure distribution of each wing section is evaluated by using XFOIL and then linearly interpolated to find the pressure over the whole wing. This information is then used for the coupling between aerodynamic and structure; the aerodynamic pressure is integrated on each element of the finite element (FE) model describing the skin of the wing, and each resulting force vector is subdivided on the nodes of the corresponding structural element. This approach guarantees that the forces and moments resulting from the aerodynamic pressure are equal to the sum 


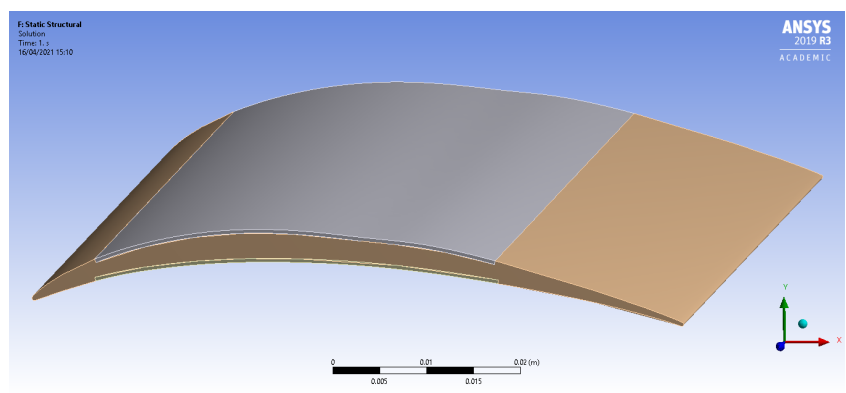

(a) Upper side

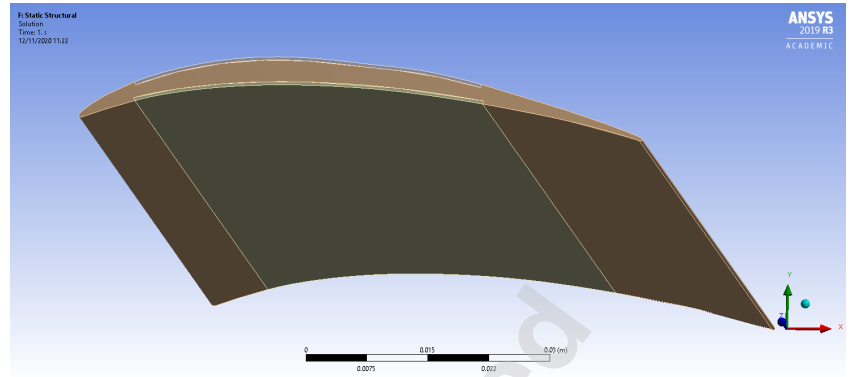

(b) Lower side

Fig. 1. Blade geometry of Case 1 with actuators on the upper and lower sides.

of nodal forces and moments in the structural model. Once the FE model of the wing is analyzed, the resulting displacements caused by both actuation and aerodynamic loads are used to define the new aerodynamic shape of the wing. The loop is repeated until convergence is achieved.

The previous works listed above study pure 3D or 2D morphing problems meaning that the same geometry is used in both structural and aerodynamic models. However, there are some situations where it is necessary to couple a two-dimensional problem with a three-dimensional one, and therefore, two different geometries need to be generated. This usually happens in the preliminary phase of a complex study, like the aero-structural analysis of aeronautical engine blades. For example in the present work, a blade cascade performance study is conducted and a 2D-3D aero-structural coupling approach is developed to link a simplified 2D CFD analysis with a 3D finite element analysis (FEA). The choice of considering a 2D aerodynamic problem has the purpose of simplifying the process and reducing the computational cost; but on the other hand, this assumption makes the aero-structural coupling more challenging because it requires the development of a specific method to match the 2D mesh (CFD) with the 3D one (FEA). This mesh matching is necessary to transfer the aerodynamic loads from the CFD analysis to the structural one. In the following sections, a detailed description of the aero-structural coupling method applied to a morphing blade study will be presented. In particular, Sec. 2 provides a general description of the steps of the developed aero-structural coupling, and some details about geometry generation, structural analysis, aerodynamic analysis, and mesh matching process. In Sec. 3, it is possible to find the results of the application of the developed coupling strategy to a morphing blade cascade study. Finally in the conclusions (Sec. 4), the work presented in this paper is summarized, and the important results are analyzed.

\section{AERO-STRUCTURAL COUPLING STRATEGY}

As mentioned in Sec. 1, the present work describes a 2D-3D aero-structural coupling strategy applied to a morphing blade cascade study. The blade geometry is morphed by using two actuators made by shape memory alloy (SMA) placed on the pressure and suction sides of the blade (Fig. 1). Once activated, they modify the leading-edge shape by bending the blade such to increase or decrease the actual angle of attack (Fig. 2). This causes a change in the flow conditions, thereby leading to a possible performance enhancement. More details about the SMA actuators and their characteristics will be provided in the following sections.

A schematic representation of the aero-structural coupling method is shown in Fig. 3. The 


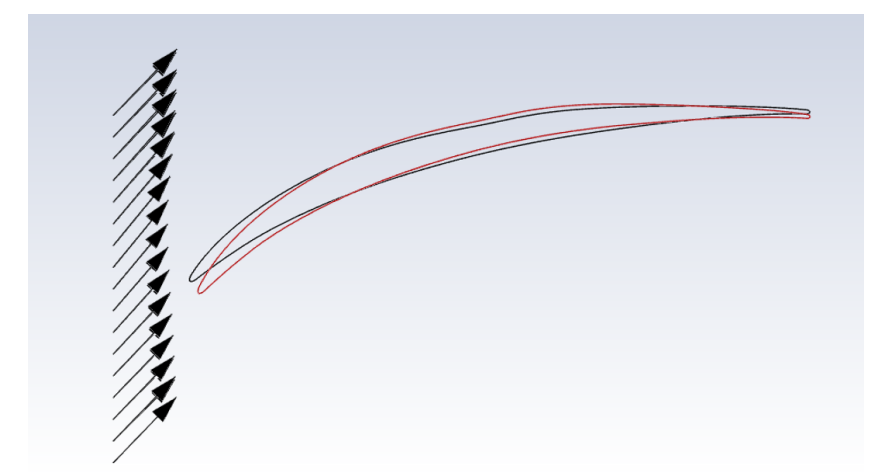

Fig. 2. Baseline blade (black) and deformed shape (red) due to the SMA actuator on the blade pressure side.

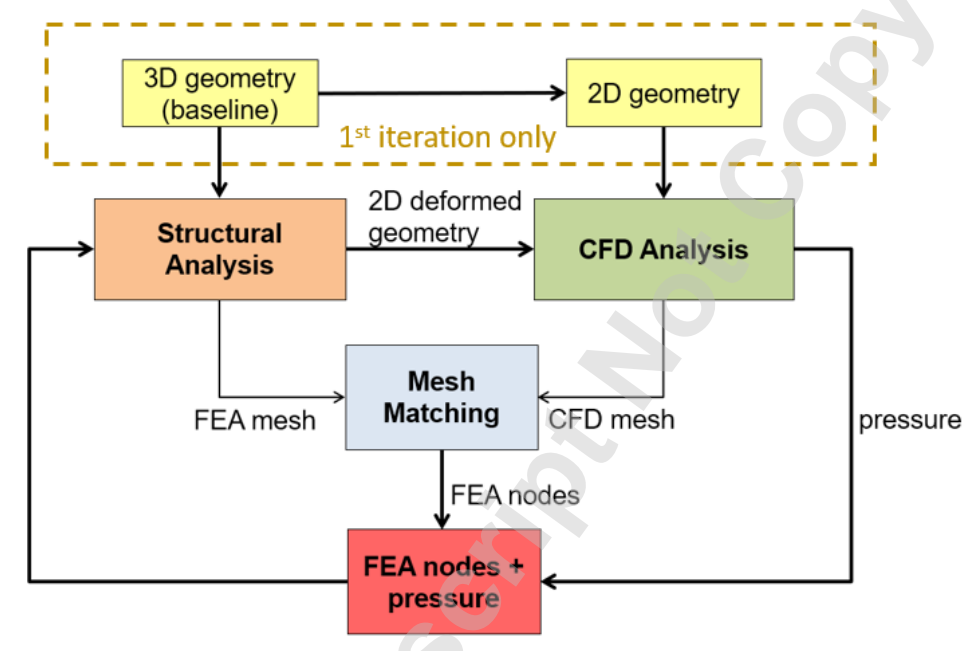

Fig. 3. Aero-structural coupling strategy scheme.

process starts with the geometry generation of the 3D baseline blade with the two actuators on the upper and lower sides (Fig. 1); this 3D geometry and the original 2D airfoil shape are used in the structural and aerodynamic analyses, respectively. The main contribution of this work lies in the "mesh matching" block in Fig. 3 which is the main element in the aero-structural coupling and it is the most challenging part in the whole process. In the mesh matching phase, the 3D structural mesh nodes coming from the FEA analysis are matched with the 2D CFD ones such that the aerodynamic loads associated to the CFD mesh nodes can be transferred to the structural ones. Once the mesh matching is completed, the "matched" FEA nodes with the associated aerodynamic loads are transferred to the structural analysis which returns both the deformed blade geometry and the new mesh as output. The previous steps are then repeated again in an iterative process that stops once a convergence criterion is satisfied. In the following sections, more details about the coupling strategy developed in this work are given.

\subsection{Geometry Generation}

As mentioned in Sec. 2, a 3D blade is generated for the structural model, and from it, the corresponding 2D airfoil is considered as the 2D geometry used in the 2D CFD analysis. Four 


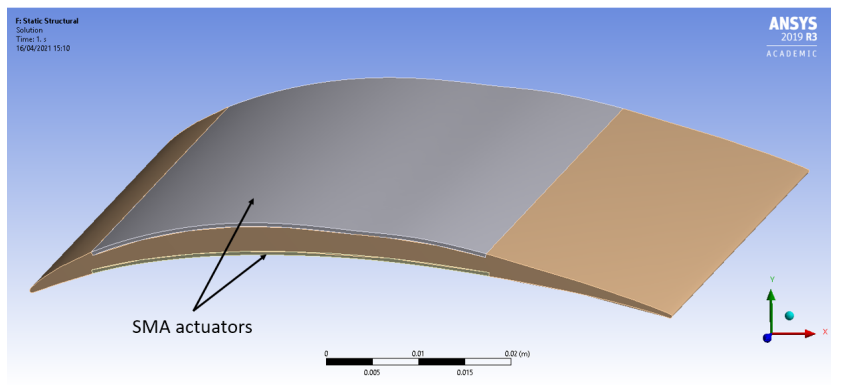

(a) Case 1

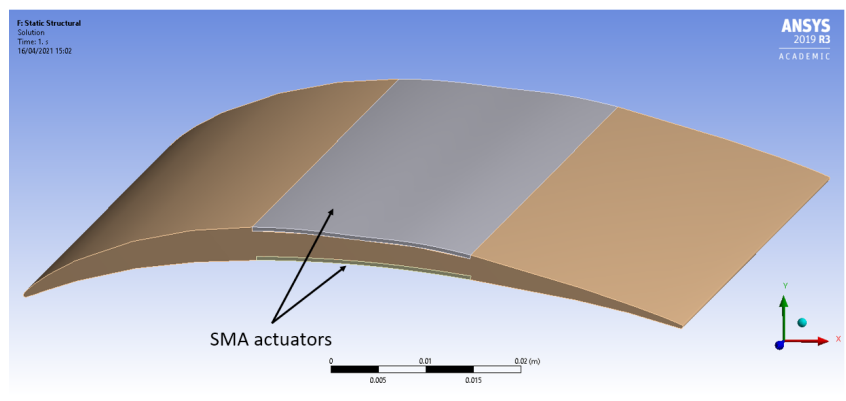

(c) Case 3

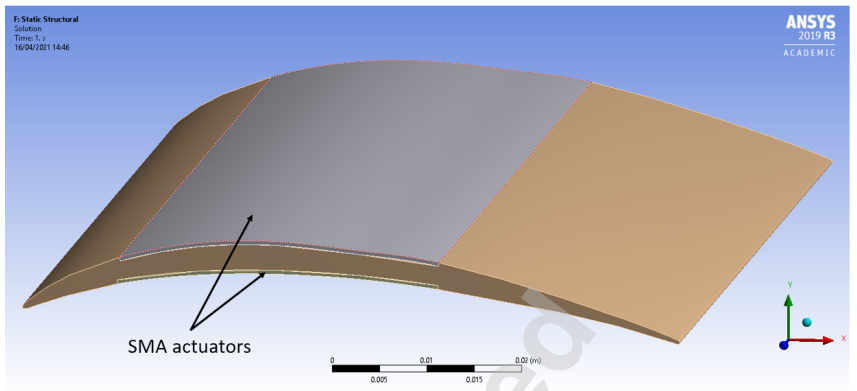

(b) Case 2

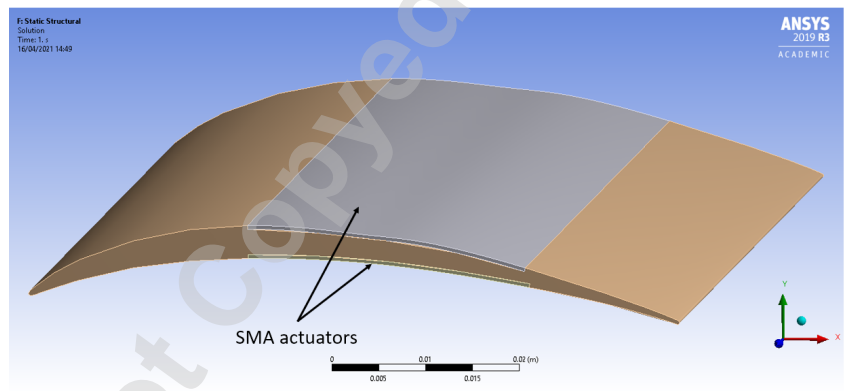

(d) Case 4

Fig. 4. Four blade configurations.

blade configurations are considered, each one has two actuators made of shape memory alloys, one is placed on the pressure side and the other on the suction side such that depending on which one is activated, the blade can bend downwards or upwards. The two actuators have the same dimensions with a thickness of $0.4 \mathrm{~mm}$; but the four blades have different starting and ending positions of the two actuators, as shown in Fig. 4 and in Tab. 1 where the starting and ending $\mathrm{x}$-coordinates are expressed in terms of percentage of chord. The choice of using SMA actuators comes from a preliminary study where also piezoelectric actuators were considered. The results of this preliminary work shows that the latter option is not able to provide the desired leading-edge displacement needed for the purpose of the present study; therefore, the final choice falls on the SMA actuators.

The baseline blade geometry is generated starting from the DLR SC14-067 [20, 21] airfoil coordinates provided by the Institute of Propulsion Technology - Department of Fan and Compressor of the German Aerospace Center (DLR). The blade is straight with no twist (Fig. 1) with an airfoil chord of $70 \mathrm{~mm}$ and a blade span of $120 \mathrm{~mm}$. The two actuators are placed inside two slots on the pressure and suction sides of the blade with a height equal to the actuator thickness $(0.4 \mathrm{~mm})$.

The 2D geometry for the CFD analysis is generated by considering the mid-span section of the $3 \mathrm{D}$ blade. According to the data provided by the Institute of Propulsion Technology (DLR), the cascade solidity $s / c$ is 0.55 , where $s$ is the cascade pitch and $c$ is the chord length. The airfoil is rotated such that the stagger angle $\beta_{S}$ is $107.37^{\circ}$, as shown in Fig. 5. Based on that, the fluid domain is drawn considering the inlet at a distance of $2 s$ from the leading-edge of the airfoil, and the outlet at $5 s$ from the trailing-edge of the airfoil (Fig. 6). 
Table 1. Actuator starting and ending $\mathrm{x}$-coordinates in percentage of chord for the four blade configurations.

\begin{tabular}{ccc}
\hline Blade & $x_{\text {start }}[\% \mathrm{c}]$ & $x_{\text {end }}[\% \mathrm{c}]$ \\
\hline Case 1 & 10 & 71 \\
Case 2 & 15 & 64 \\
Case 3 & 35 & 68 \\
Case 4 & 34 & 76 \\
\hline
\end{tabular}

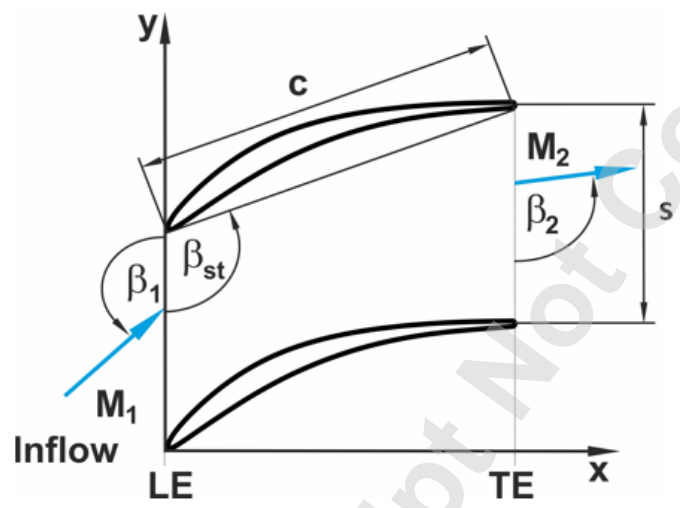

Fig. 5. Airfoil cascade.

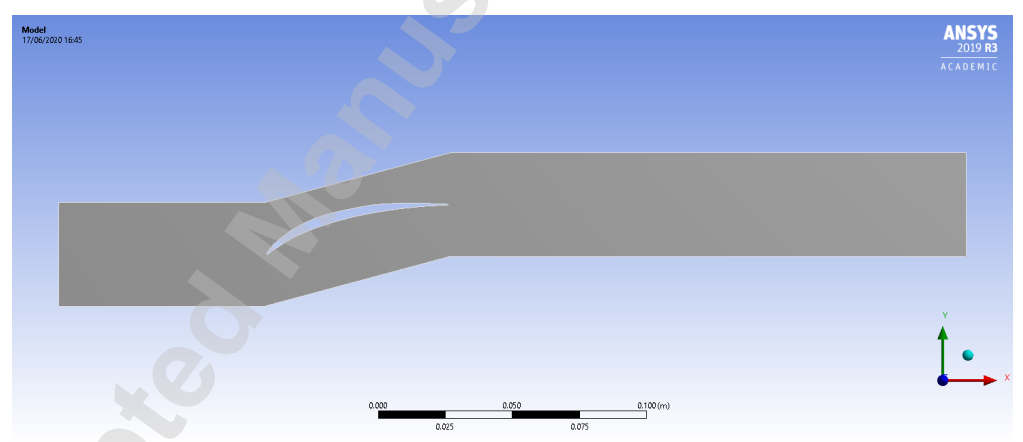

Fig. 6. 2D geometry with fluid domain.

\subsection{Structural Analysis}

The finite element analysis is conducted in ANSYS Workbench ${ }^{\circledR}$ and a scheme of the analysis sub-components is shown in Fig. 7.

First, the material properties (block A) of the blade and the two actuators are defined; in particular, aluminum alloy is assigned to the blade and shape memory alloy to the actuators. Since the paper focuses mainly on the aero-structural coupling, the SMA model is simplified extremely and a more detailed one will be implemented in future works. Therefore, the shape memory alloy 


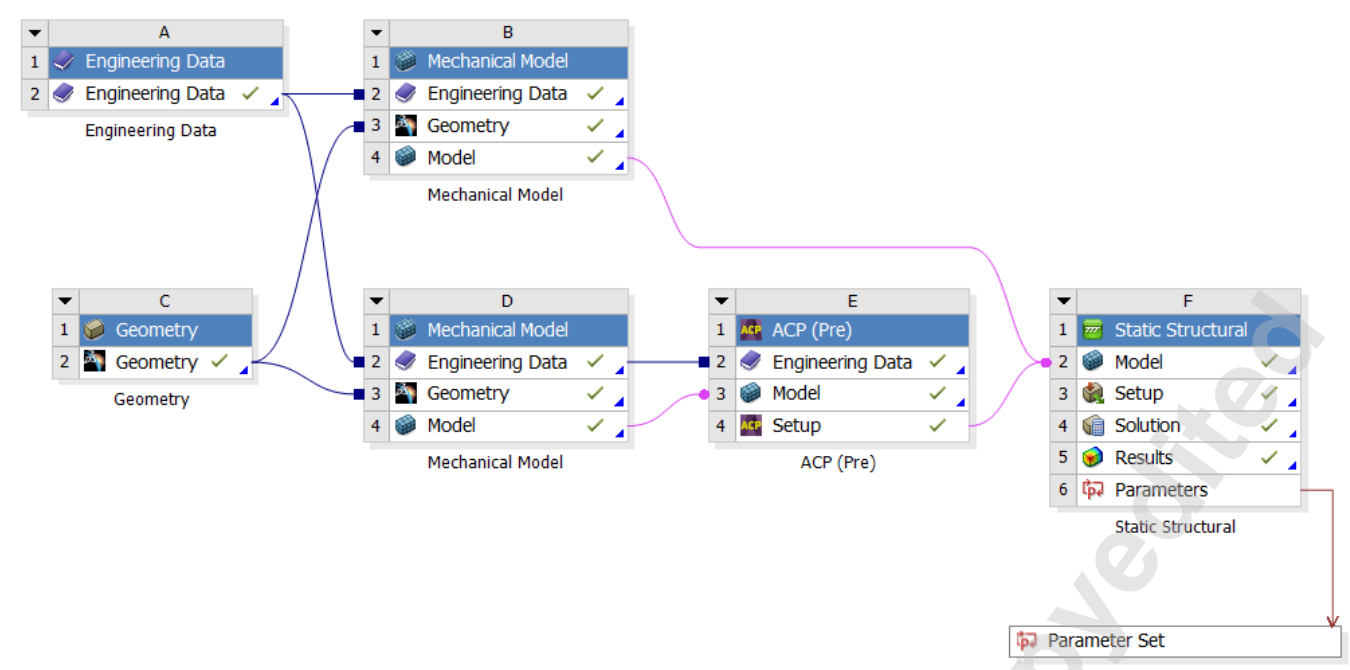

Fig. 7. Schematic representation of the steps for the structural analysis.

material is modeled as an isotropic material with linear material behavior. In this first step, even martensite and austenite stiffness as well as the hyteresis are not taken into account, and the actuation of the SMA is considered only in one direction. The Young's modulus chosen for this material is $30 \mathrm{GPa}$, and the Poisson's ratio is 0.33 , since the actuators are modeled in first approximation by assuming that they are made by $100 \%$ SMA. For such a simplified SMA model, a thermal analogy is used with a coefficient $\alpha$ of $-2 \%$ per $100^{\circ} \mathrm{C}$ temperature difference:

$$
\alpha=\frac{\epsilon}{\Delta T}=\frac{-0.02}{100}=-0.0002 /{ }^{\circ} \mathrm{C}
$$

After the material assignment, the 3D blade geometry is created (block $\mathrm{C}$ ) and imported into the blocks $B$ and $D$ where the mesh is generated for the blade and the two actuators separately. In particular, block B contains the blade geometry without the actuators and it gives in output the resulting mesh, as shown in Fig. 8. In the block $D$, the upper and lower actuators separated from the blade are modeled and their mesh is generated (Fig. 9). It is important to underline that the number of mesh nodes along the width and length of the two actuators is set equal to the number of nodes on the corresponding slots on the blade. In such a way, the two meshes (blade and actuators) can be easily superimposed and merged together (Fig. 10).

The two actuators need to be modeled with a "direction of polarization" that defines the axis along with they deform when activated. As it is possible to see in Fig. 11, the SMA material is oriented along the $\mathrm{x}$-axis (chordwise direction); this means that once a temperature is assigned to the material, the actuators contract in the x-direction as shown by the green arrows in the figure. This contraction leads to a bending of the blade in the upward direction when the actuator on the suction side is active, or downwards when the one on the pressure side is actuated, as shown in Fig. 12.

Once the deformation direction of the SMA is defined (block E), the blade and the actuators can be put together in block F (Fig. 10). At this point, the boundary conditions for the structural analysis 


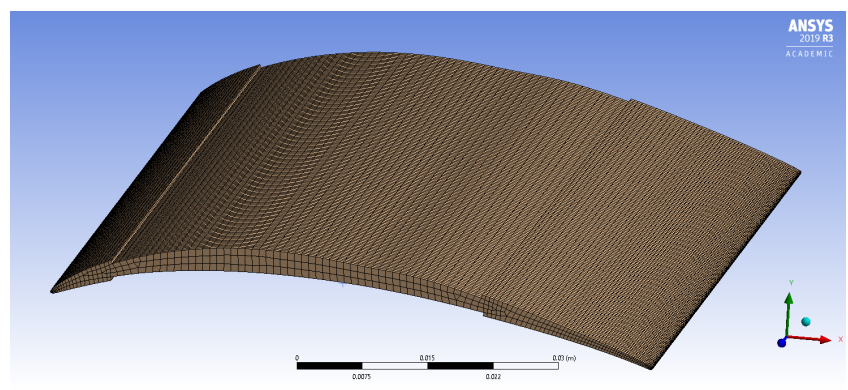

Fig. 8. Mesh of the blade without actuators.

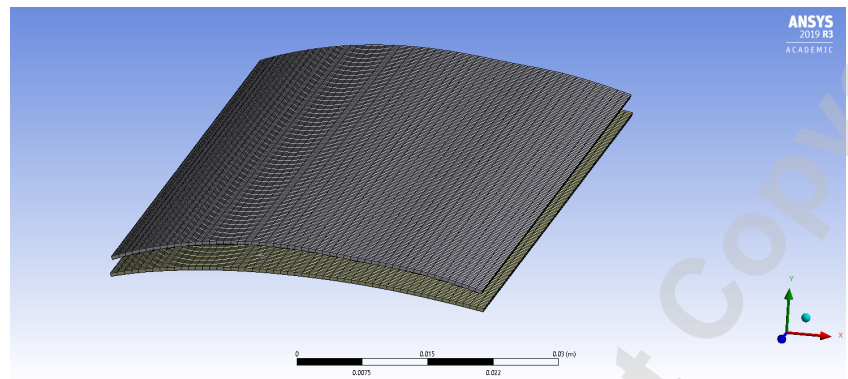

Fig. 9. Mesh of the two actuators.

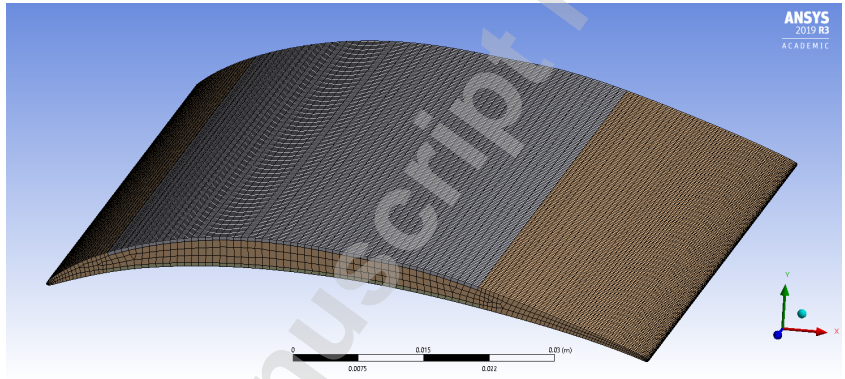

Fig. 10. Mesh of the blade with the two actuators.

are defined in terms of edge clamping and thermal condition for the actuators. It has been decided to clamp the blade on both lateral sides, but considering only the portion of the blade close to the trailing-edge and not covered by the actuators, as shown in Fig. 13. In particular remembering that the $x$-axis is along the chord and the $z$-axis along the blade span, at the coordinate $z=0$ (blade root), the three directions $x, y, z$ are set to zero (clamped); while at $z=120 \mathrm{~mm}$ (blade tip), the $z$-direction is left free not to over-constrain the blade and to avoid unwanted internal stresses. Another important parameter to set for the actuators is the thermal condition which is a temperature that based on the thermal analogy simulates the voltage on the actuator leading to its activation. For this specific work, the value assigned to the thermal condition is $100^{\circ} \mathrm{C}$ for the active actuator (Fig. 14), considering that $0^{\circ} \mathrm{C}$ corresponds to zero strain.

\subsection{Aerodynamic Analysis}

ANSYS Fluent ${ }^{\circledR}$ is used for the CFD analysis. As already mentioned, a 2D problem is considered to reduce the computational cost; therefore, the flow over a 2D airfoil cascade is simulated. As 


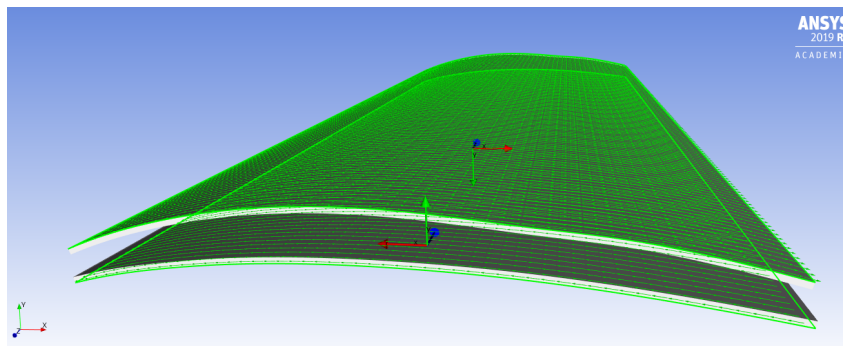

(a)

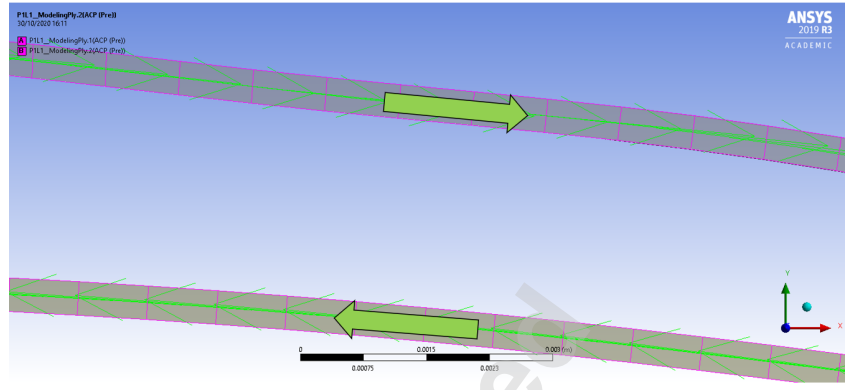

(b)

Fig. 11. Direction of deformation for the two SMA actuators.

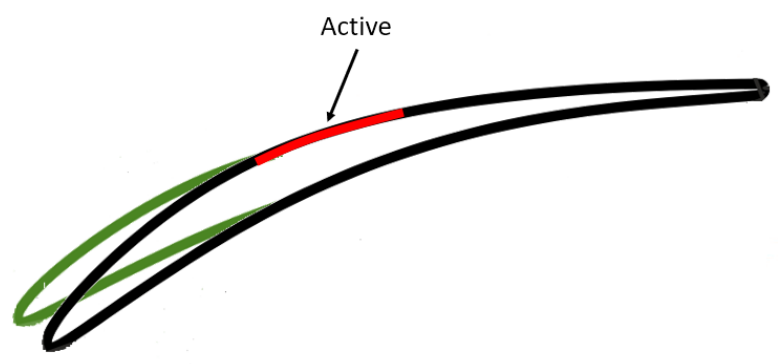

(a) Bending due to the upper actuator

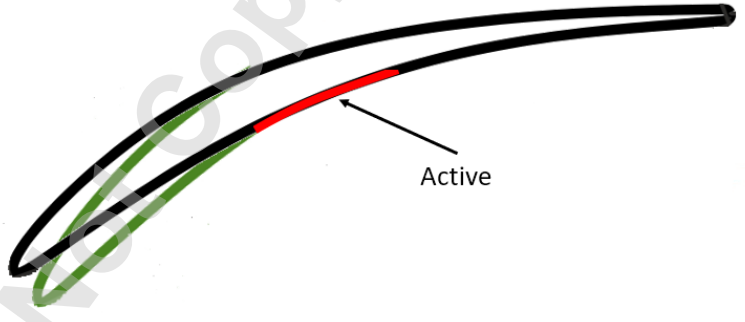

(b) Bending due to the lower actuator

Fig. 12. Blade bending directions.

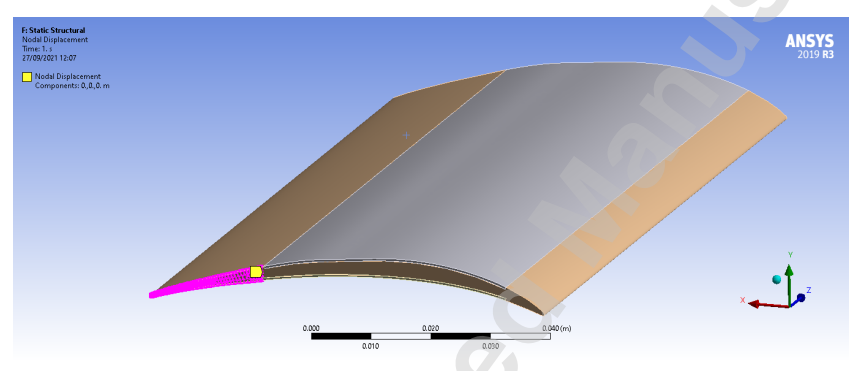

(a) Blade root

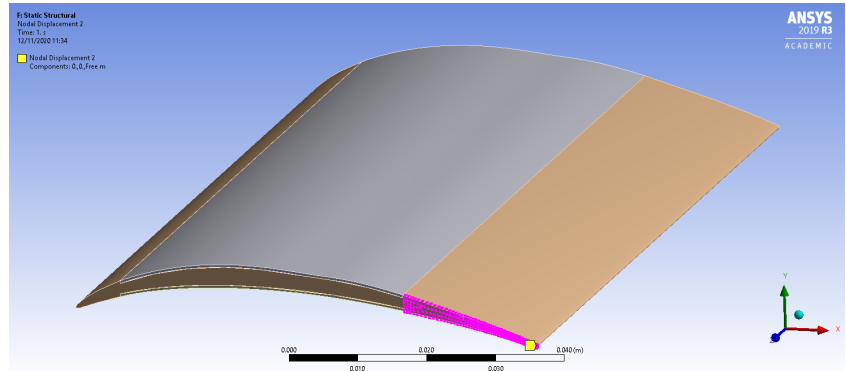

(b) Blade tip

Fig. 13. Clamped area (pink) at the lateral sides of the blade.

already described in Sec. 2.1, the mid-span section of the 3D blade is considered as 2D geometry. The inflow conditions provided by the Institute of Propulsion Technology (DLR) are characterized by an inlet Mach number $M_{1}$ of 0.65 , an inflow angle $\beta_{1}$ of $134^{\circ}$ at design condition, an inlet total temperature $T_{01}$ is of $315 \mathrm{~K}$ and an inlet total pressure $p_{01}$ is of $122500 \mathrm{~Pa}$.

Figure 15 shows the schematic representation of the steps followed in the aerodynamic analysis. The 2D mesh is generated by means of Ansys mesher tool starting from the 2D airfoil shape (Sec. 2.1). The mesh is quadrilateral with 30 prism layers close to the airfoil surface such to have 


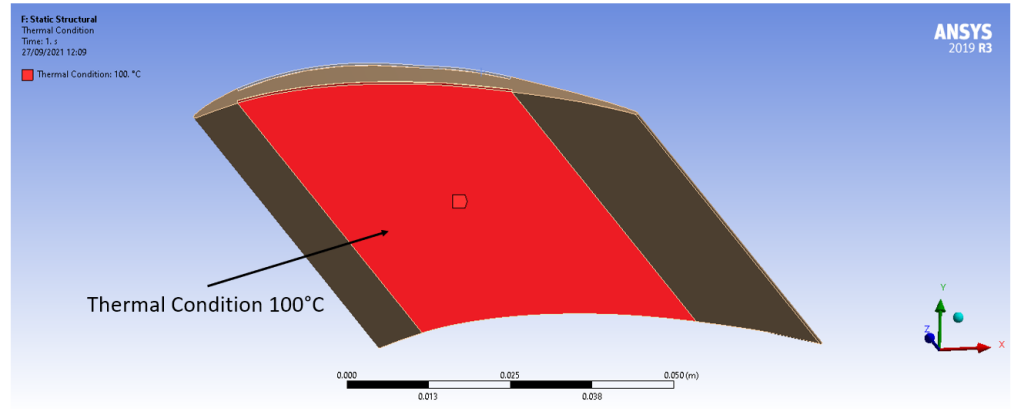

Fig. 14. Thermal condition applied to the lower side actuator.

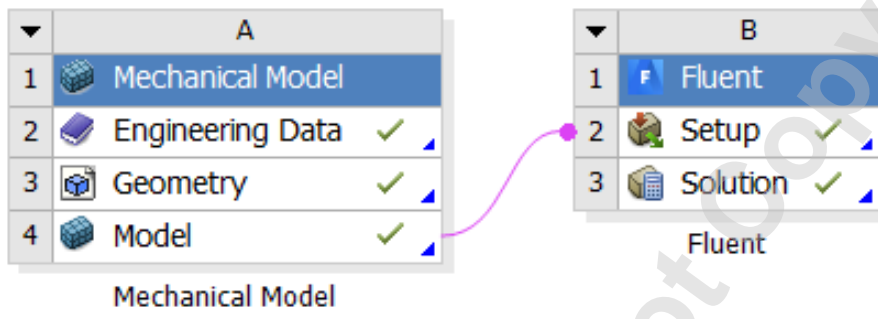

Fig. 15. Schematic representation of the steps for the CFD analysis.

a $y+\leq 1$. After a grid independence study, the final mesh results to have about 150000 elements and it takes only few seconds to be generated. Figure 16 shows the 2D mesh of the fluid domain with a closer view to the leading-edge and trailing-edge regions of the airfoil.

The boundary conditions assigned to the fluid domain are represented in Fig. 17, where the airfoil is a no-slip wall, the inlet is a pressure inlet and the outlet is a pressure outlet. The two sides above and below the airfoil are periodic boundaries to simulate the cascade.

The flow is steady, compressible and fully turbulent; the $k-\omega S S T$ turbulence model is selected and coupled with a $y+\leq 1$ close to the airfoil surface such that the entire boundary layer can be solved. The computational time for a single CFD simulation is about 10 minutes by using four processors in parallel.

To obtain reliable results, it is important to conduct a validation of the CFD results by comparing them with experimental data. For the present work, no experimental data are available but the validation is conducted by using computational results provided by the Institute of Propulsion Technology (DLR). In particular, they conducted a 3D aerodynamic analysis with an in-house CFD software and the results in terms of pressure distribution over the mid-span section airfoil are compared with 2D CFD simulations in the present work. Figure 18 shows the pressure distribution at three different inflow angles: $128^{\circ}, 134^{\circ}$ (design condition), $142^{\circ}$. As it is possible to notice, the $2 \mathrm{D}$ CFD results of the present work (orange dots) match quite well the provided data when the inflow angle is less or equal to the design value, i.e. when the flow is mostly attached and without any separation. On the other side when the inflow angle increases above the design condition, a flow separation starts and the 2D CFD data does not seem to perfectly match the reference one. This is more evident when the flow separation becomes predominant at $142^{\circ}$ of inflow angle (orange 


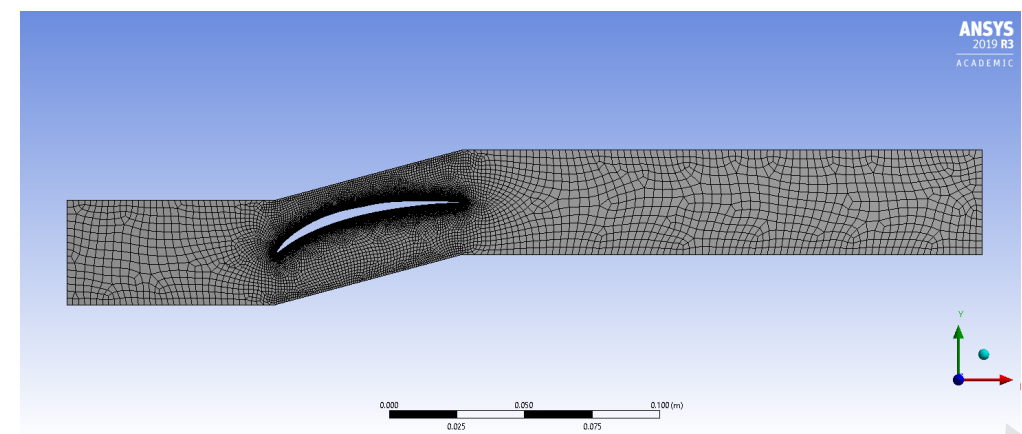

(a)

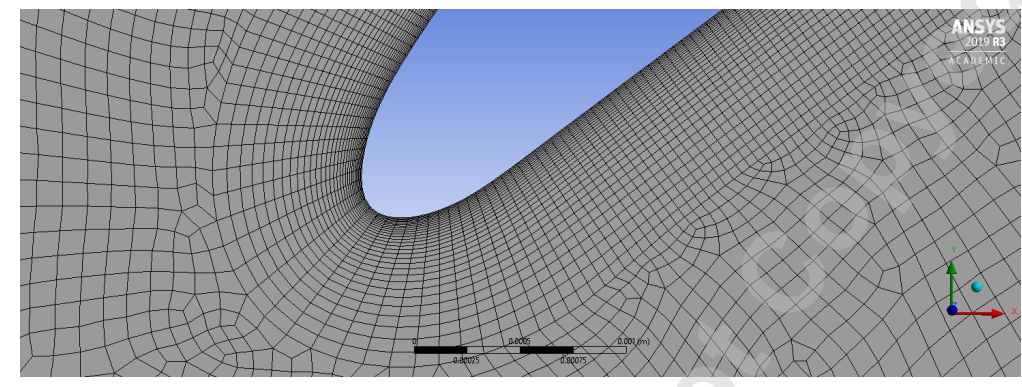

(b)

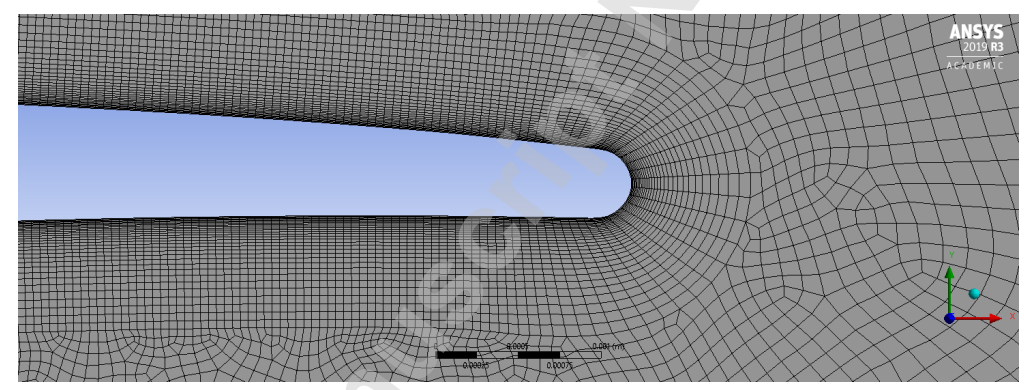

(c)

Fig. 16. CFD mesh with detailed view on the leading and trailing edges.

dots in Fig. 18). However by reducing the inflow angle up to $136^{\circ}$ (green dots) in the $2 \mathrm{D}$ analysis, it is possible to notice that the 2D CFD data matches the reference one at $142^{\circ}$. The reason of that is because the reference data comes from 3D CFD simulations of a compressor cascade where the blades are attached to the wall at both root and tip, and therefore, the three-dimensional flow effects are captured in the solution. The interaction between the boundary layer on the blade surface and walls leads to secondary flows that create blockage and affect the cascade performance. Such a three dimensional effect becomes more relevant at high inflow angles when the flow over the blade starts to separate. The blockage effect described above explains the reason why a lower inflow angle $\left(136^{\circ}\right)$ in the 2D CFD analysis matches the given 3D CFD data at $142^{\circ}$.

The two outputs of the aerodynamic analysis which are 2D mesh and pressure distribution over the airfoil are saved and used for the fluid-structure coupling as described in the following sections. 


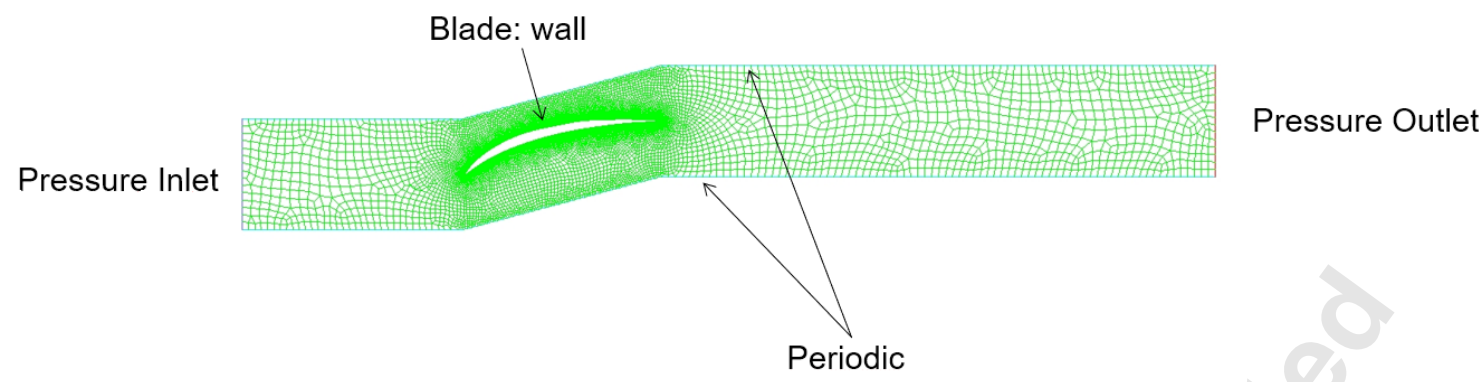

Fig. 17. CFD boundary conditions.

\subsection{Mesh Matching}

The mesh matching step is the most important in the coupling process presented in this paper. It is also the most challenging one since it was not possible to use the integrated tools available in ANSYS Workbench ${ }^{\circledR}$ for the aero-structural coupling due to the 2D-3D problem. In fact, the ANSYS coupling tools and other previous methods developed in the past require the same geometry for both aerodynamic and structural analyses. In the present work, the coupling between the two disciplines involves two different geometries: 3D for the structural problem, 2D for the CFD analysis. Therefore, the difficulty is to build a tool able to match a 3D mesh with a 2D one. In Fig. 19, there is the schematic representation of the coupling process described so far; no aerodynamic loads are transferred yet and the geometry is the baseline one without any deformation. At this point, the "mesh matching" box takes the structural mesh (3D) and the CFD mesh (2D) as input and it returns the FEA mesh nodes with the associated static pressure resulting from the CFD analysis.

More in detail, the structural mesh nodes represented by the black dots in Fig. 20 are filtered such to select only the mid-span section nodes represented by the green dots. At this point, another filtering process is necessary to get only the structural mesh nodes on the surface of the mid-span section (black dots in Fig. 21) to compare them with the CFD ones. Once the filtered mid-span section FEA nodes are obtained, the distances between those nodes and the CFD ones can be calculated. Therefore, it is possible to identify the mid-section FEA nodes closest to each CFD mesh airfoil node, and then transfer the computed CFD pressure to the corresponding FEA mesh nodes. Figure 22 summarizes the steps of the process described above where the inputs are the 2D coordinates of both structural mesh nodes (already filtered) and aerodynamic mesh nodes; the output is given by the 2D coordinates of the matched FEA mesh coupled with the associated static pressure. These two elements (2D FEA mesh nodes and pressure) are used inside a new structural analysis to find the deformed blade configuration due to both aerodynamic loads and actuation of the morphing device (Fig. 23).

\subsection{Structural Analysis with Aerodynamic Loads}

As mentioned in Sec. 2.4, the aerodynamic loads associated to the 2D filtered structural mesh nodes must be uploaded into the structural analysis, and to do that, the "External Data" tool in ANSYS Workbench ${ }^{\circledR}$ is used, as shown in Fig. 24. The External Data block is connected to the Static Structural one such that the data can be transferred in the structural analysis. The uploaded pressure distribution on the blade mid-span section (Fig. 25(a)) is projected over the whole span (Fig. 25(b),25(c)). Even though a constant pressure distribution along the blade span is not real- 
istic, it is important to remember that the work presented here wants to be a simplified approach for the development and implementation of a 2D-3D aero-structural coupling method; therefore, some assumptions are necessary in this preliminary phase.

Once the aerodynamic loads are uploaded into the structural analysis, one of the two actuators is activated and the resulting deformed geometry due to both aerodynamic loads and actuation is found. Figure 26 shows an example of the output geometry after the run of the structural analysis with loads; particularly evident is the comparison between the deformed geometry (colorful blade) and the original one (gray blade). In this example, the blade shows an exaggerated deformation only to have a better visualization of the deformed geometry due to aerodynamic loads and actuation. Figure 26 shows also the corresponding 2D geometry derived from the whole 3D deformed blade by adding the $x$ and $y$ displacement resulting from the structural analysis with loads to the baseline coordinates of the mid-span blade section. Figure 27 shows also a comparison in terms of total deformation of the same blade under the influence of only the actuator force (Fig. 27(a)), only the aerodynamic loads (Fig. 27(b)), and the combination of the two effects (Fig. 27(c)). As it is possible to see, the bending resulting by the activation of the actuator is able to strongly oppose the deformation due to the aerodynamic loads.

Similarly, the deformed structural mesh used in the following mesh matching step is obtained by adding the nodal displacements found in the structural analysis with loads to the original baseline mesh. After that, the matching process between the deformed FEA and CFD meshes is repeated as described in the previous section (Sec. 2.4), and the loop between aerodynamic and structural analyses continues until the convergence criterion is satisfied. As detailed described in the following section, the convergence is reached when the difference in the leading-edge displacement along the $y$-axis between two consecutive iterations is less than a given tolerance value. In the following section, some results about the application of the aero-structural coupling method described so far are reported.

\section{RESULTS}

In this section, the convergence of the aero-structural coupling loop is shown for the four blade configurations described in Sec. 2.1 at an inflow angle $\beta_{1}$ of $136^{\circ}$ (off-design condition). As already mentioned, the aero-structural coupling process is iterative and it stops when the difference in the leading-edge displacement along the $y$-axis between two consecutive iterations is less than $2 \cdot 10^{-5}$ $[\mathrm{m}]$ :

$$
\Delta y=y_{i}-y_{i-1}<2 \cdot 10^{-5}
$$

Figure 28 shows the value of $\Delta y$ as function of the iteration counter for the four blade configurations, and as it is possible to notice, all the cases simulated show a convergence after up to four iterations.

Once the convergence of the fluid-structure interaction framework has been empirically proved, it is interesting to see how the resulting deformation at the leading edge affects the cascade performance evaluated in terms of total pressure losses. The total pressure loss coefficient $\omega$ is defined as: 


$$
\omega=\frac{p_{01}-\bar{p}_{02}}{q_{1}}
$$

where $p_{01}$ is total pressure at inlet, $\bar{p}_{02}$ is the average total pressure at outlet, and $q_{1}$ is the dynamic pressure at inlet. A reduction of $\omega$ means a reduction in the pressure losses, and therefore, an enhancement in the cascade aerodynamic performance. First of all, it is important to investigate if the blade needs to be bent in the upward or downward direction in order to improve the aerodynamic characteristics of the flow and therefore the cascade performance in off-design condition. As a consequence, it will be clear which one of the two actuators has to be activated. In the specific case of the present work based on the inflow conditions and on the baseline geometry, the blade must be bent downwards to achieve a performance improvement; therefore, the actuator on the pressure side of the blade must be activated. Based on that, the thermal condition of $100^{\circ} \mathrm{C}$ in the structural analysis is applied only to the lower side actuator (Sec. 2.2). It is important to remind that the cascade is working in off-design condition $\left(\beta_{1}=136^{\circ}\right)$, and therefore, the flow over the airfoil is characterized by a strong separation that affects the residuals of the CFD analysis. Indeed as shown in Fig. 29(a), the residuals in the baseline CFD simulation do not converge due to a strong flow separation on the suction side of the blade (Fig. 30(a)), and they are characterized by strong oscillations. Even though the authors know that the strong oscillations in the baseline CFD simulation could be a concern for the final results, the purpose of the paper is not the deep investigation of the separation phenomena. Moreover, the baseline value of $\omega$ resulting from the 2D CFD analysis which is $0.09 \pm 0.1$ is in good agreement with the reference value of the 3D CFD simulations which is 0.102 . The final deformed blade instead shows a stability and a convergence in the CFD residuals due to a reduction in the flow separation (Fig. 30) given by the leading-edge morphing. In particular, Tab. 2 shows the comparison of the inlet metal angle $\kappa_{1}$ (Fig. 31) between the baseline and the morphed blades; Tab. 3 lists the y-displacement, and the pressure loss reduction achieved by each single blade configuration thanks to the activation of the SMA actuator on the pressure side of the blade. Such a displacement is very small but it is able to strongly affect the inlet blade metal angle and the overall performance of the cascade, as it is possible to notice in Tab. 2 and Tab. 3. In particular, Case 1 shows a final y-displacement of $1.46 \mathrm{~mm}$, an increase of the inlet metal angle up to $51^{\circ}$, and a total pressure loss coefficient of 0.042 which compared to the oscillating baseline value of $0.09 \pm 0.1$, it results to be decreased of about $53 \%$.

Another parameter commonly used to characterize the performance of compressor cascades is the deviation angle $\delta$; therefore, it is interesting to see the effects of morphing on this performance parameter. The deviation angle is the difference between the outlet flow angle $\beta_{2}$ and the outlet blade metal angle $\kappa_{2}$ (Fig. 31): a reduction of the deviation angle implies an increase in the flow turning and a higher pressure rise. Table 4 lists the values of the deviation angle for the baseline blade and the four deformed geometries: all the morphed blades show a relevant decrease in the deviation compared to the baseline.

\section{CONCLUSION}

In the present work, an aero-structural coupling strategy has been developed and applied to a morphing blade cascade study. In particular, the idea of using a 2D CFD solver aims to reduce the problem complexity and consequently the overall computational cost required by the analysis. On 
Table 2. Inlet blade metal angle.

\begin{tabular}{cc}
\hline Blade & $\kappa_{1}[\mathrm{deg}]$ \\
\hline Baseline & 46.8793 \\
Case 1 & 51.0544 \\
Case 2 & 50.8264 \\
Case 3 & 49.8559 \\
Case 4 & 48.5690 \\
\hline
\end{tabular}

Table 3. Y-displacement, total pressure loss coefficient, and pressure loss reduction.

\begin{tabular}{cccc}
\hline Blade & $y[\mathrm{~m}]$ & $\omega$ & $\omega$ reduction [\%] \\
\hline Case 1 & -0.00146 & 0.042414 & 53 \\
Case 2 & -0.00138 & 0.044742 & 50 \\
Case 3 & -0.00104 & 0.045852 & 49 \\
Case 4 & -0.00059 & 0.044827 & 50 \\
\hline
\end{tabular}

Table 4. Deviation angle comparison.

\begin{tabular}{cc}
\hline Blade & $\delta[\mathrm{deg}]$ \\
\hline Baseline & 9.5931 \\
Case 1 & 5.8151 \\
Case 2 & 6.8187 \\
Case 3 & 6.4193 \\
Case 4 & 4.5224 \\
\hline
\end{tabular}

the other side, this simplification introduces a challenge in the development of the aero-structural coupling method; in particular, it is necessary to formulate a procedure able to match a 3D structural mesh with a 2D aerodynamic one. The mesh matching step consists in the conversion of the $3 \mathrm{D}$ structural mesh into a 2D one by filtering the mesh nodes and considering only the ones on the mid-span section. At this point, the filtered 2D FEA mesh can be compared with the CFD one, and the distances between FEA and CFD nodes can be calculated to find the closest CFD mesh nodes to the 2D structural ones. The corresponding static pressure computed by the aerodynamic 
analysis and originally assigned to the CFD mesh nodes, is then transferred to the "matched" FEA mesh nodes. The new load configuration of the structural analysis aims to obtain the deformed blade geometry due to both aerodynamic loads and the activation of the SMA actuator used to morph the leading-edge. Four different blade configurations in terms of dimensions and position of the actuators have been simulated, and the iterative loop between aerodynamics and structural mechanics shows a convergence in all the cases. Once demonstrated the reliability of the approach, the developed aero-structural coupling tool has been applied to the performance study of a morphing blade cascade. The results of this applied problem show that even with a small leading-edge displacement (less than $2 \mathrm{~mm}$ ) due to actuation of the SMA actuator, it is possible to reach a cascade performance enhancement of about $53 \%$ in off-design condition. The work presented in this paper is the first step of a more complex study involving aero-structural coupling and multidisciplinary optimization applied to a morphing blade cascade.

\section{NOMENCLATURE}

A Aspect ratio

$c$ Airfoil chord

$C_{L} \quad$ Lift coefficient

$M_{1} \quad$ Inlet Mach number

$p_{01} \quad$ Inlet total pressure

$\bar{p}_{02}$ Average total pressure at outlet

$s$ Cascade pitch

$s / c$ Cascade solidity

$T_{01}$ Inlet total temperature

$q_{1}$ Dynamic pressure at inlet

$y \quad$ Displacement along the $y$-axis

$\alpha$ Thermal coefficient

$\beta_{S} \quad$ Stagger angle

$\beta_{1}$ Inlet flow angle

$\beta_{2} \quad$ Outlet flow angle

$\delta$ Deviation angle

$\kappa_{1} \quad$ Inlet blade metal angle

$\kappa_{2} \quad$ Outlet blade metal angle

$\omega$ Total pressure loss coefficient

\section{REFERENCES}

[1] Vincent, J., 2000. "Smart by name, smart by nature". Smart materials and structures, 9(3), p. 255.

[2] Mueller, T., and Lawerenz, M., 2003. "Shape adaptive airfoils for turbomachinery applications undergoing large deformations". In 44th AIAA/ASME/ASCE/AHS/ASC Structures, Structural Dynamics, and Materials Conference, p. 1561.

[3] Garcia, E., 2002. "Smart structures and actuators: past, present, and future". In Smart Structures and Materials 2002: Industrial and Commercial Applications of Smart Structures Technologies, Vol. 4698, International Society for Optics and Photonics, pp. 1-12. 
[4] Chopra, I., 2002. "Review of state of art of smart structures and integrated systems". AIAA journal, 40(11), pp. 2145-2187.

[5] Thirupathi, S. R., Seshu, P., and Naganathan, N. G., 1997. "A finite-element static analysis of smart turbine blades". Smart materials and structures, 6(5), p. 607.

[6] Cross, C. J., and Fleeter, S., 2002. "Shunted piezoelectrics for passive control of turbomachine blading flow-induced vibrations". Smart materials and Structures, 11(2), p. 239.

[7] Pern, N., and Jacob, J., 1999. "Aerodynamic flow control using shape adaptive surfaces". In Proceeding of, Citeseer, pp. 12-15.

[8] Riemenschneider, J., Huxdorf, O., and Opitz, S., 2014. "Effects of piezoceramic actuator in quasistatic use". In Smart Materials, Adaptive Structures and Intelligent Systems, Vol. 46148, American Society of Mechanical Engineers, p. V001T01A013.

[9] Monner, H. P., Huxdorf, O., Riemenschneider, J., and Keimer, R., 2015. "Design and manufacturing of morphing fan blades for experimental investigations in a cascaded wind tunnel". In 23rd AIAA/AHS Adaptive Structures Conference, p. 0790.

[10] Loewy, R. G., 1997. "Recent developments in smart structures with aeronautical applications". Smart Materials and Structures, 6(5), p. R11.

[11] Bil, C., Massey, K., and Abdullah, E. J., 2013. "Wing morphing control with shape memory alloy actuators". Journal of Intelligent Material Systems and Structures, 24(7), pp. 879-898.

[12] Barbarino, S., Flores, E. S., Ajaj, R. M., Dayyani, I., and Friswell, M. I., 2014. "A review on shape memory alloys with applications to morphing aircraft". Smart materials and structures, 23(6), p. 063001.

[13] Ko, S.-H., Bae, J.-S., and Rho, J.-H., 2014. "Development of a morphing flap using shape memory alloy actuators: the aerodynamic characteristics of a morphing flap". Smart materials and structures, 23(7), p. 074015.

[14] Ricci, S., and Terraneo, M., 2006. "Application of mdo techniques to the preliminary design of morphed aircraft". In 11th AIAA/ISSMO Multidisciplinary Analysis and Optimization Conference, p. 7018.

[15] Potsdam, M., Yeo, H., and Johnson, W., 2006. "Rotor airloads prediction using loose aerodynamic/structural coupling". Journal of Aircraft, 43(3), pp. 732-742.

[16] Kroll, N., Heinrich, R., Krueger, W., and Nagel, B., 2008. "Fluid-structure coupling for aerodynamic analysis and design: a dlr perspective". In 46th AIAA Aerospace Sciences Meeting and Exhibit, p. 561.

[17] Gamboa, P., Vale, J., Lau, F., and Suleman, A., 2009. "Optimization of a morphing wing based on coupled aerodynamic and structural constraints". AIAA journal, 47(9), pp. 2087-2104.

[18] Molinari, G., Quack, M., Dmitriev, V., Morari, M., Jenny, P., and Ermanni, P., 2011. "Aerostructural optimization of morphing airfoils for adaptive wings". Journal of Intelligent Material Systems and Structures, 22(10), pp. 1075-1089.

[19] Molinari, G., Arrieta, A. F., and Ermanni, P., 2014. "Aero-structural optimization of threedimensional adaptive wings with embedded smart actuators". AIAA journal, 52(9), pp. 19401951.

[20] Reutter, O., Hemmert-Pottmann, S., Hergt, A., and Nicke, E., 2014. "Endwall contouring and fillet design for reducing losses and homogenizing the outflow of a compressor cascade". In Turbo Expo: Power for Land, Sea, and Air, Vol. 45608, American Society of Mechanical Engineers, p. V02AT37A007.

[21] Reutter, O., Rozanski, M., Hergt, A., and Nicke, E., 2017. "Advanced endwall contouring for loss reduction and outflow homogenization for an optimized compressor cascade". Interna- 
tional journal of turbomachinery, Propulsion and Power, 2(1), p. 1. 


\section{LIST OF FIGURES}

1 Blade geometry of Case 1 with actuators on the upper and lower sides. . . . . . . . .

2 Baseline blade (black) and deformed shape (red) due to the shape memory alloy (SMA) actuator on the blade pressure side. . . . . . . . . . . . . . . . 5

3 Aero-structural coupling strategy scheme. . . . . . . . . . . . . . . 5

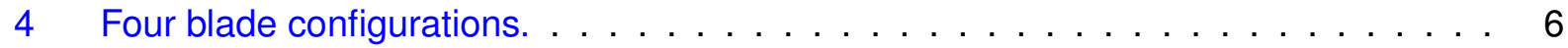

5 Airfoil cascade. . . . . . . . . . . . . . . . . . . . . 7

$62 \mathrm{D}$ geometry with fluid domain. . . . . . . . . . . . . . . . . 7

7 Schematic representation of the steps for the structural analysis. . . . . . . . . . . 8

8 Mesh of the blade without actuators. . . . . . . . . . . . . . . 9

9 Mesh of the two actuators. . . . . . . . . . . . . . . . . . . . . 9

10 Mesh of the blade with the two actuators. . . . . . . . . . . . . . . 9

11 Direction of deformation for the two shape memory alloy (SMA) actuators. . . . . . 10

12 Blade bending directions. . . . . . . . . . . . . . . . . . . . 10

13 Clamped area (pink) at the lateral sides of the blade. . . . . . . . . . . . . . . . 10

14 Thermal condition applied to the lower side actuator. . . . . . . . . . . . . . . . 11

15 Schematic representation of the steps for the CFD analysis. . . . . . . . . . . . . . 11

16 CFD mesh with detailed view on the leading and trailing edges. . . . . . . . . . . . 12

17 CFD boundary conditions. . . . . . . . . . . . . . . . . . . . 13

18 CFD result comparison with reference data at an inflow angle of $128^{\circ}, 134^{\circ}$ and $142^{\circ} .22$

19 Schematic representation of the coupling process at the first stage when no aerodynamic loads are involved. . . . . . . . . . . . . . . . . . 23

20 Mesh nodes of the 3D blade. . . . . . . . . . . . . . . . . . . 23

21 Mesh nodes on the mid-span section. . . . . . . . . . . . . . . . . . . 24

22 Mesh matching steps. . . . . . . . . . . . . . . . . . . . . . . . . . 24

23 Schematic representation of the coupling process with the aerodynamic loads. . . . 25

24 Aerodynamic loads imported into the structural analysis through the External Data tool. . . . . . . . . . . . . . . . . . . . . . . . 25

25 Same pressure distribution in the CFD and FEA analyses. . . . . . . . . . . 26

26 Resulting 3D and 2D deformed geometries. . . . . . . . . . . . . . . . 27

27 Deformation comparison. . . . . . . . . . . . . . . . . . . . 28

28 Iterations in the aero-structural coupling loop for the four different blade configurations. 28

29 CFD residuals of the baseline blade and final deformed blade (Case 1). . . . . . . . 29

30 Total pressure distribution of the baseline blade and final deformed blade (Case 1). . 30

31 Angles in a compressor cascade. . . . . . . . . . . . . . . . . . 31 


\section{LIST OF TABLES}

1 Actuator starting and ending $x$-coordinates in percentage of chord for the four blade configurations. . . . . . . . . . . . . . . . . . 7

2 Inlet blade metal angle. . . . . . . . . . . . . . . . . . 16

$3 \quad$ Y-displacement, total pressure loss coefficient, and pressure loss reduction. . . . . . 16

4 Deviation angle comparison. . . . . . . . . . . . . . . . . . . 16 


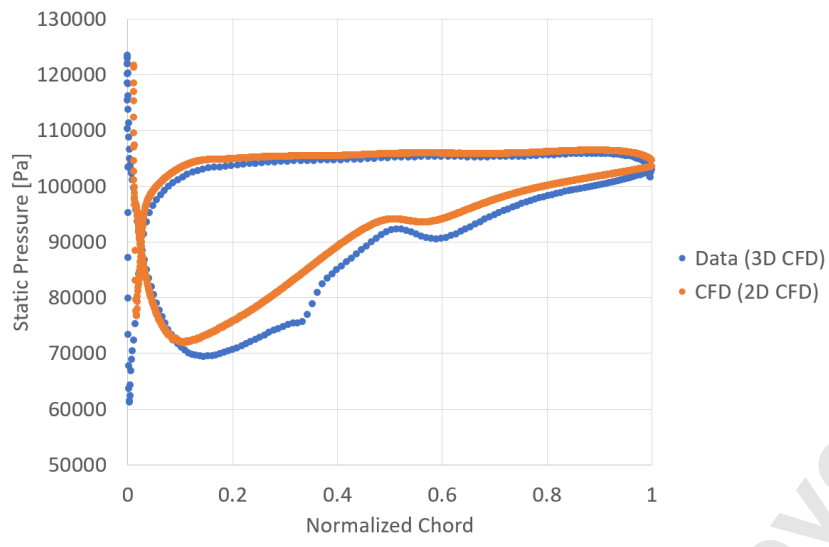

(a) $\beta_{1}=128^{\circ}$

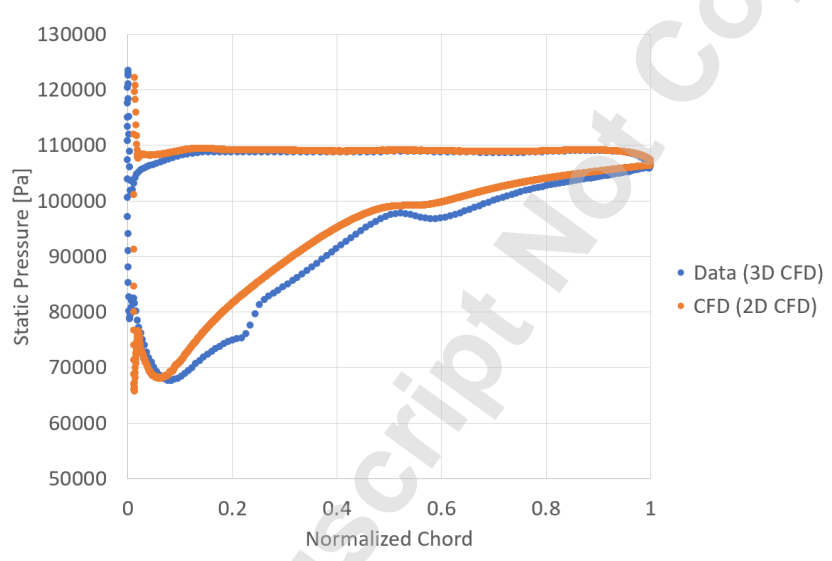

(b) $\beta_{1}=134^{\circ}$

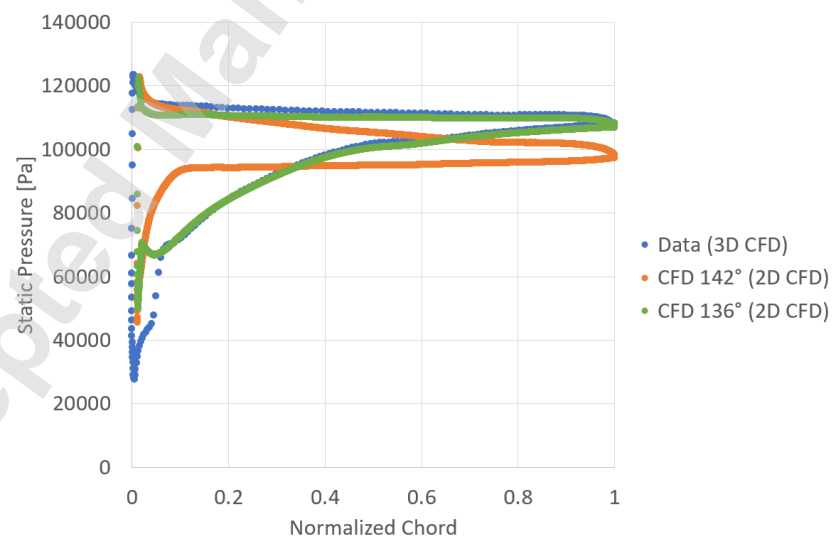

(c) $\beta_{1}=142^{\circ}$

Fig. 18. CFD result comparison with reference data at an inflow angle of $128^{\circ}, 134^{\circ}$ and $142^{\circ}$. 


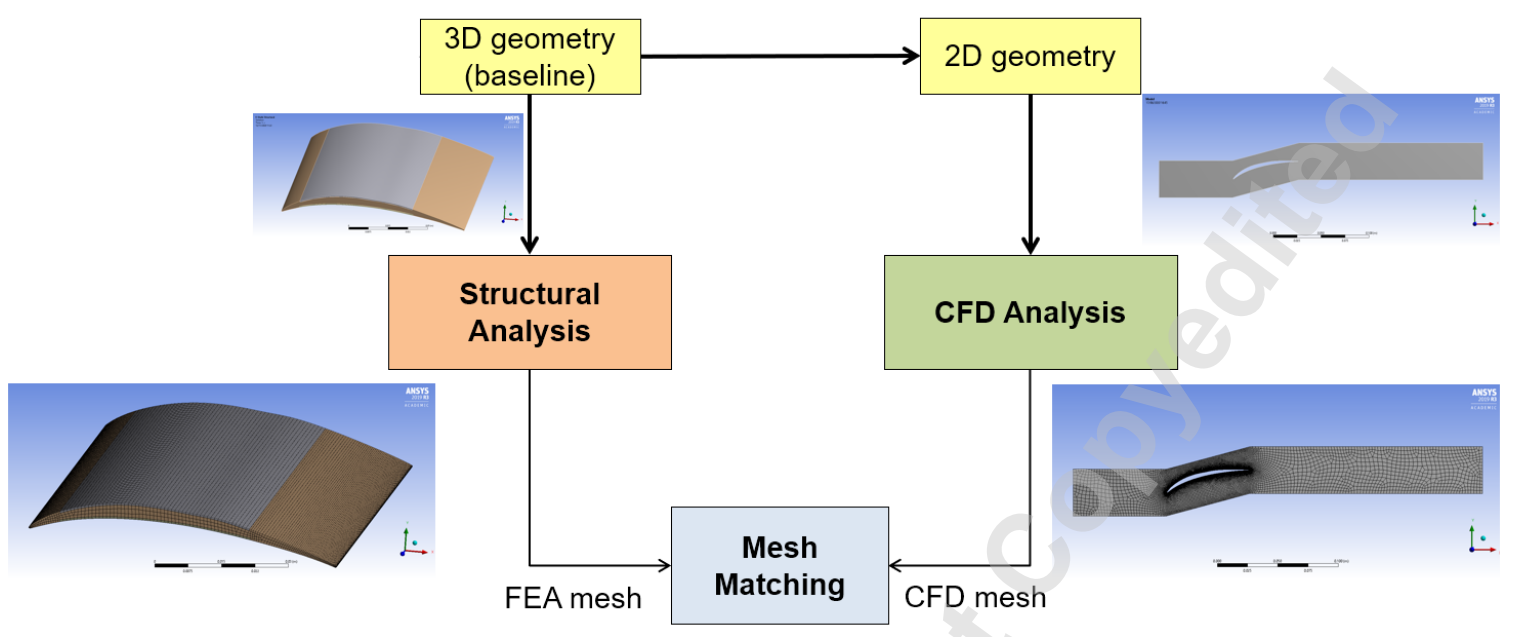

Fig. 19. Schematic representation of the coupling process at the first stage when no aerodynamic loads are involved.

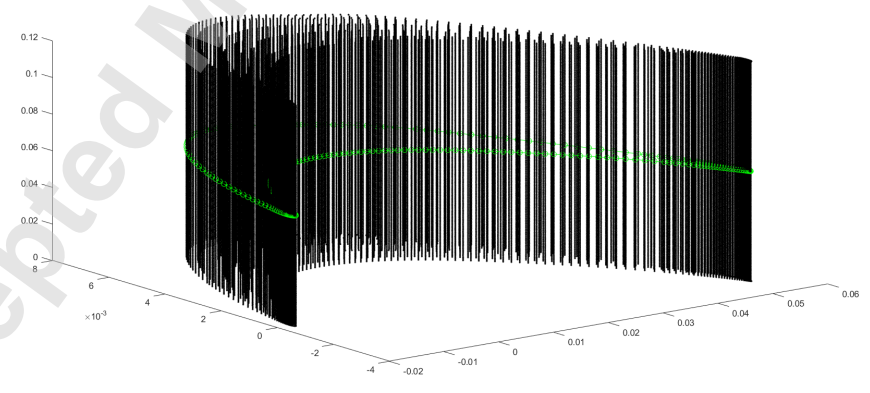

Fig. 20. Mesh nodes of the 3D blade. 


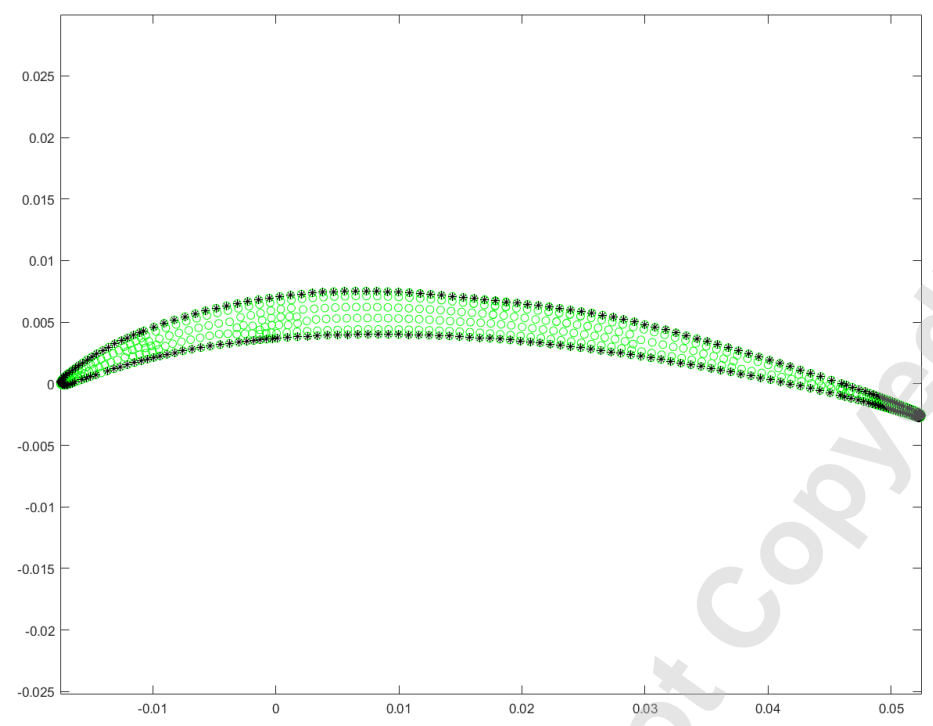

Fig. 21. Mesh nodes on the mid-span section.

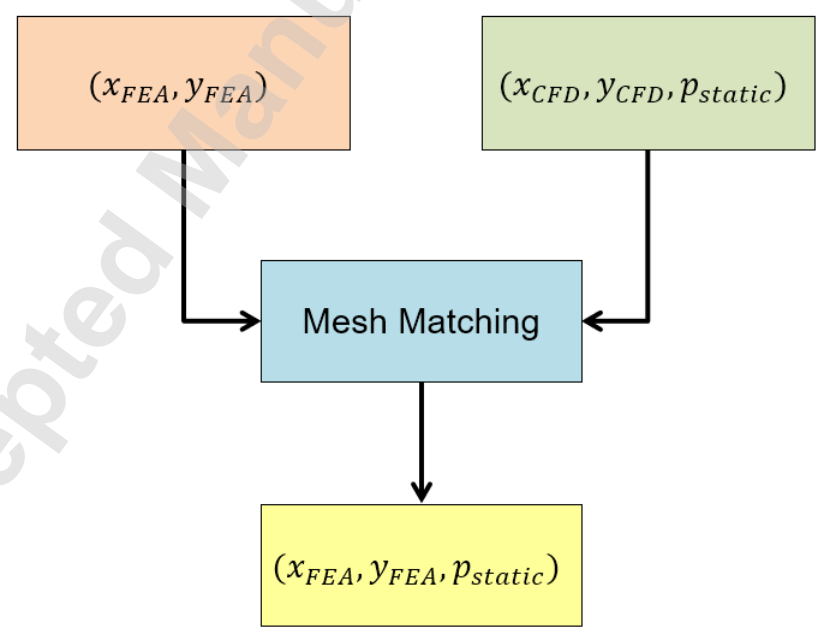

Fig. 22. Mesh matching steps. 


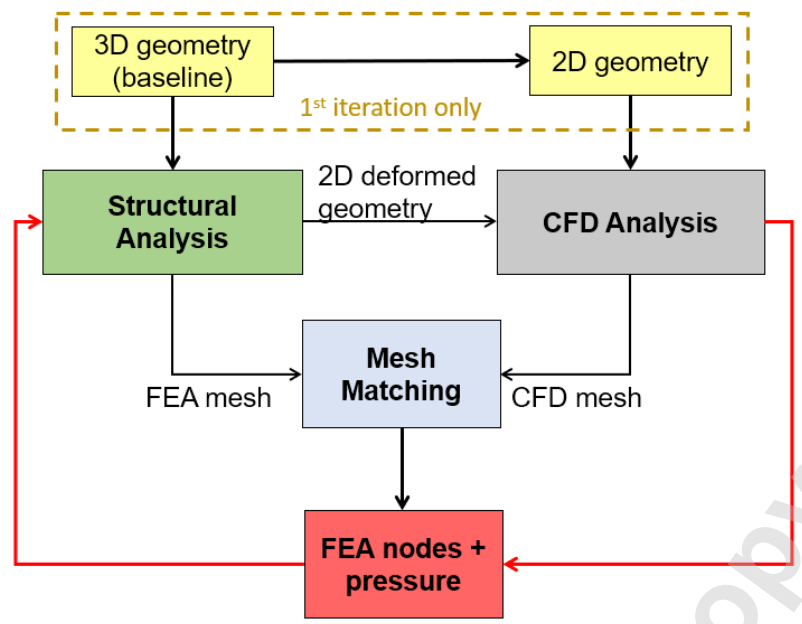

Fig. 23. Schematic representation of the coupling process with the aerodynamic loads.

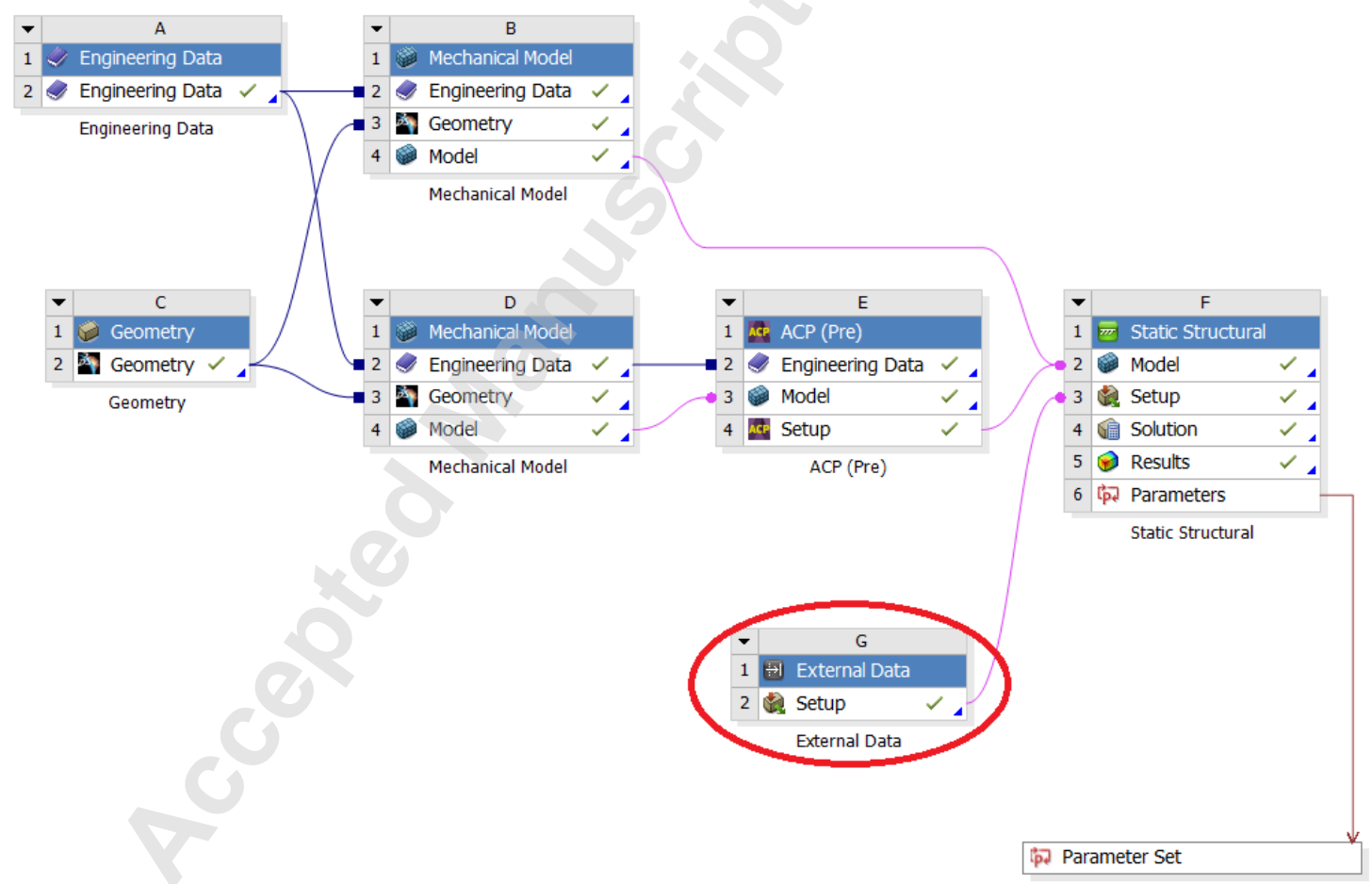

Fig. 24. Aerodynamic loads imported into the structural analysis through the External Data tool. 


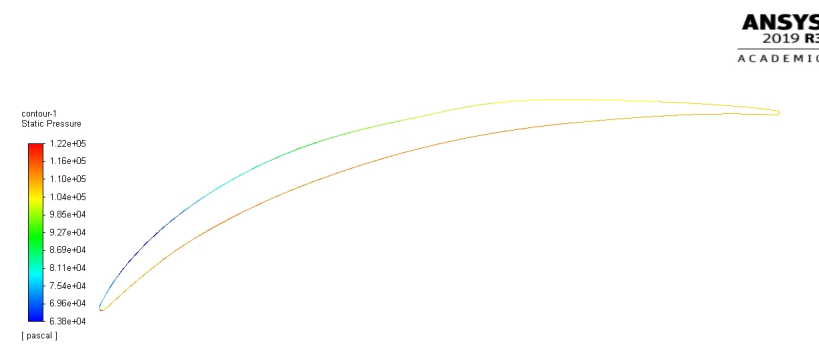

(a) 2D airfoil

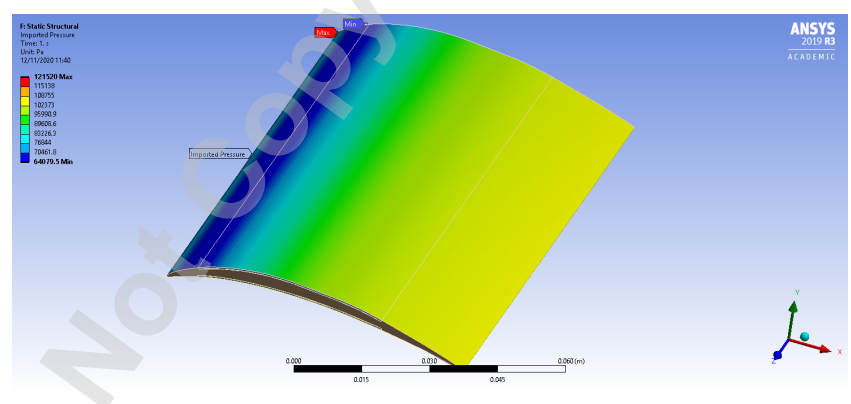

(b) Blade suction side

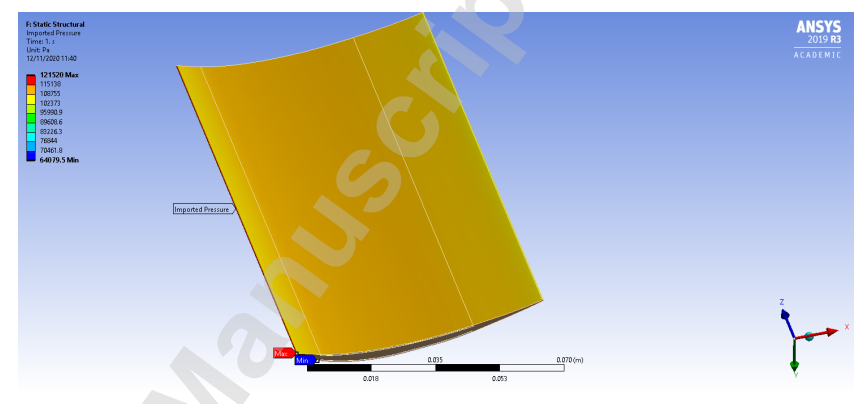

(c) Blade pressure side

Fig. 25. Same pressure distribution in the CFD and FEA analyses. 


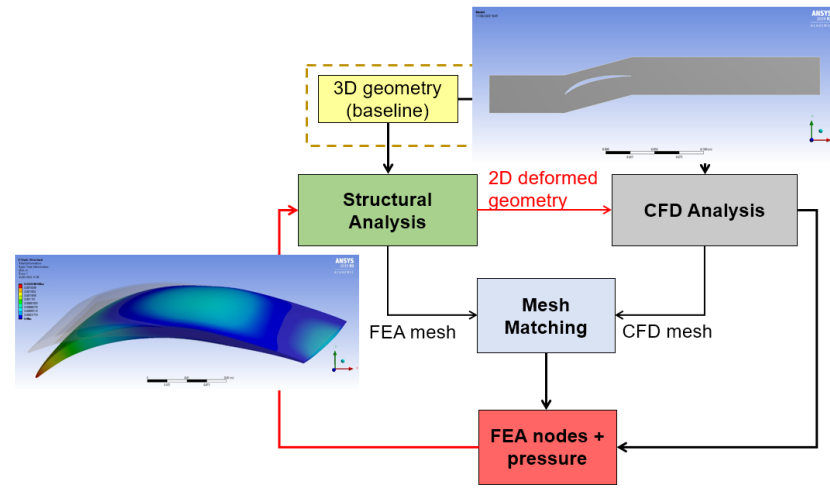

(a) Schematic representation of the structural analysis output

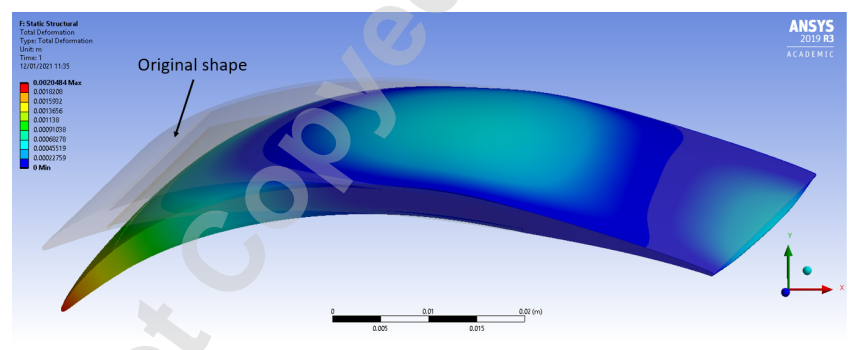

(b) 3D baseline-deformed geometry comparison

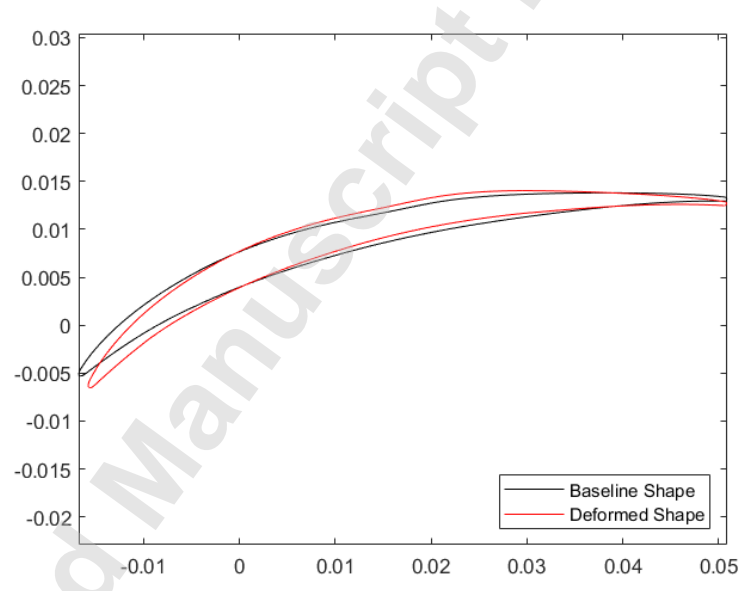

(c) 2D baseline-deformed geometry comparison

Fig. 26. Resulting 3D and 2D deformed geometries. 


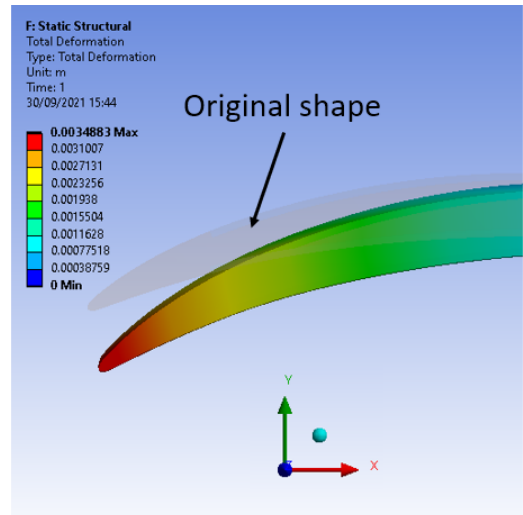

(a) Only actuator

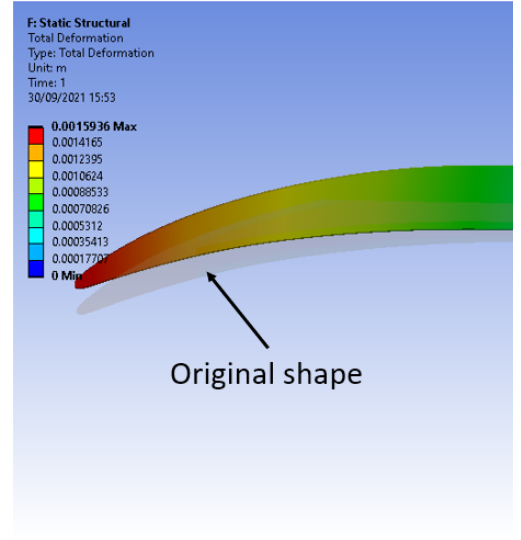

(b) Only aerodynamic loads

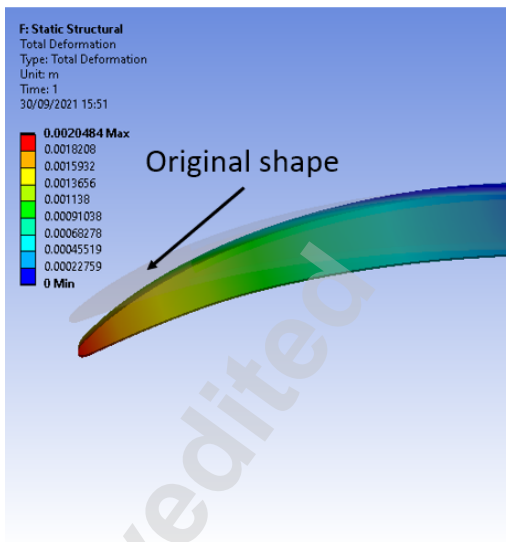

(c) Combination of aero loads and actuator

Fig. 27. Deformation comparison.

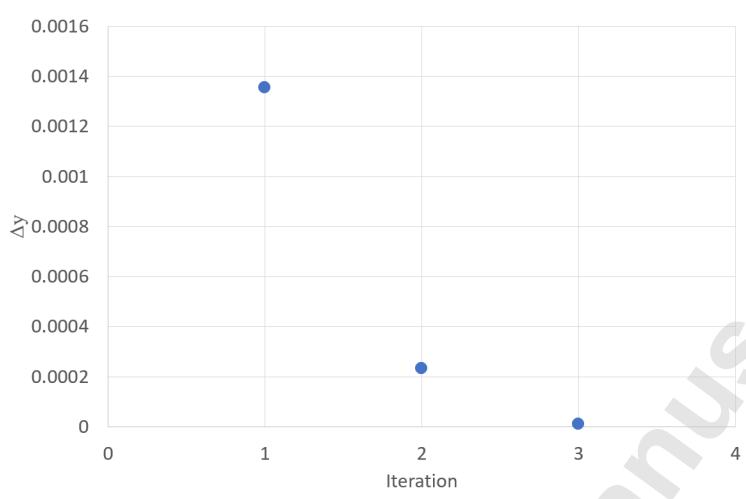

(a) Case 1

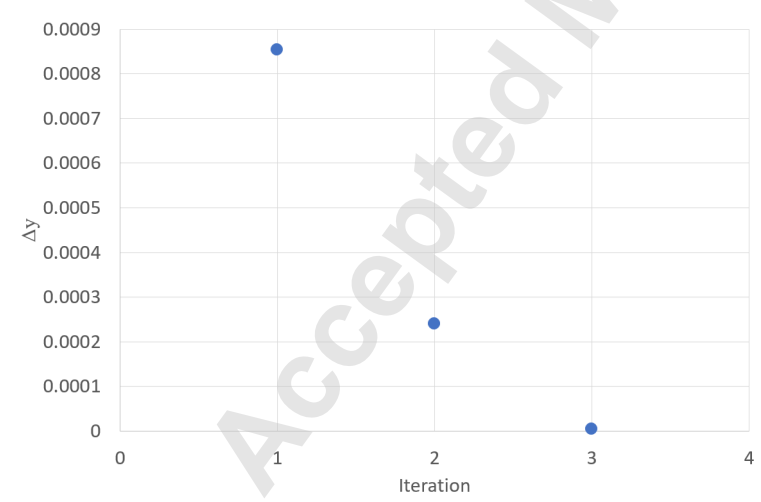

(c) Case 3

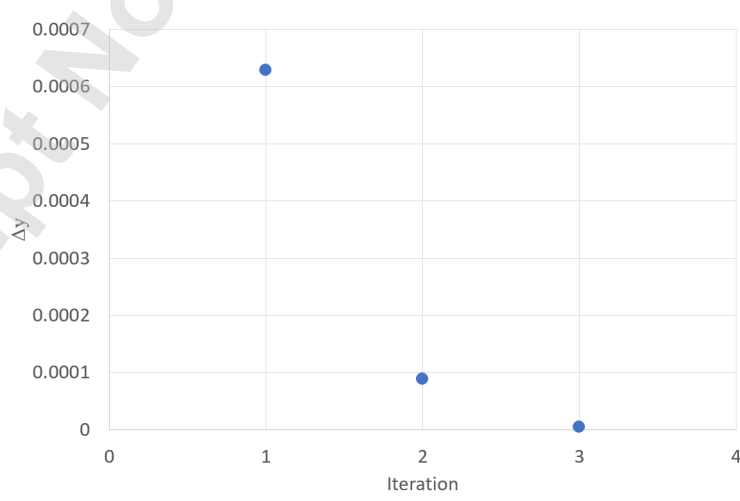

(b) Case 2

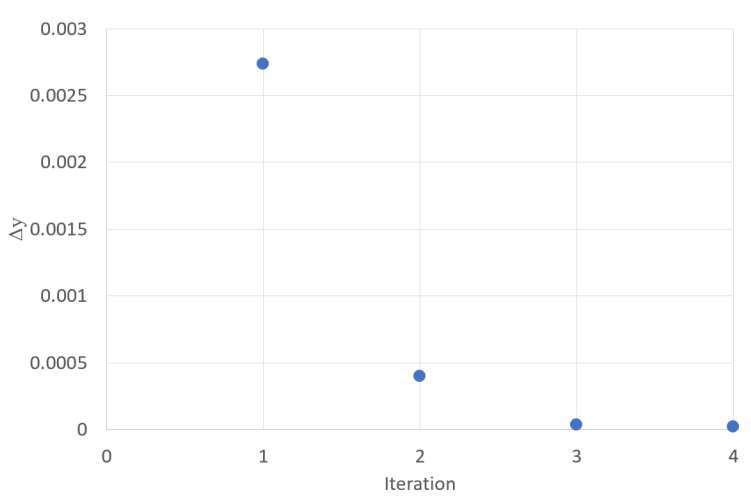

(d) Case 4

Fig. 28. Iterations in the aero-structural coupling loop for the four different blade configurations. 


\begin{tabular}{|c|}
\hline Residuals \\
\hline continuity \\
\hline x-velocity \\
\hline y-velocity \\
\hline energy \\
\hline $\mathrm{k}$ \\
\hline omega \\
\hline
\end{tabular}

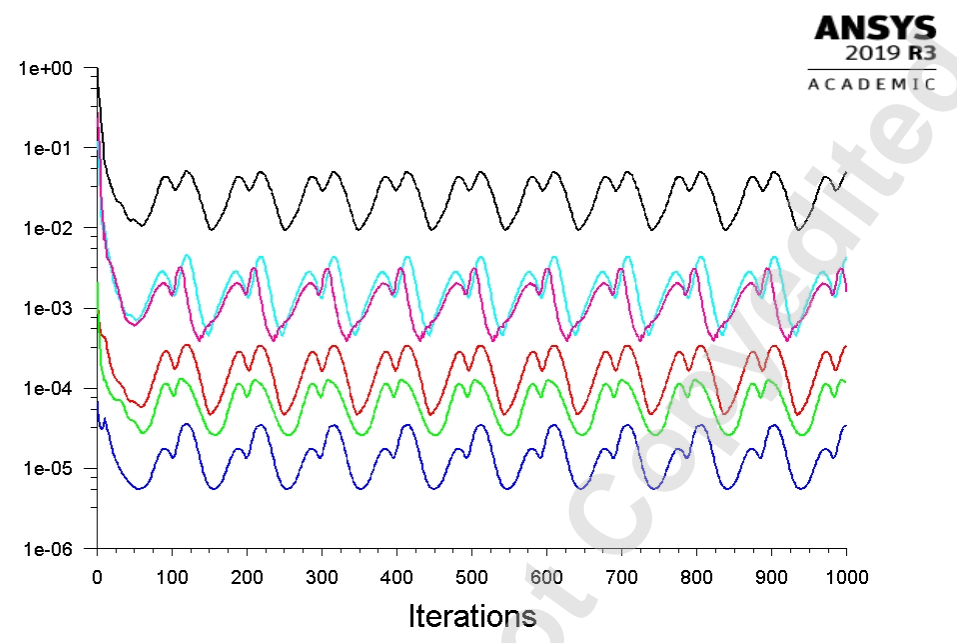

(a) Baseline

\begin{tabular}{|c|}
\hline Residuals \\
\hline continuity \\
\hline x-velocity \\
\hline y-velocity \\
\hline energy \\
\hline $\mathrm{k}$ \\
\hline omega \\
\hline
\end{tabular}

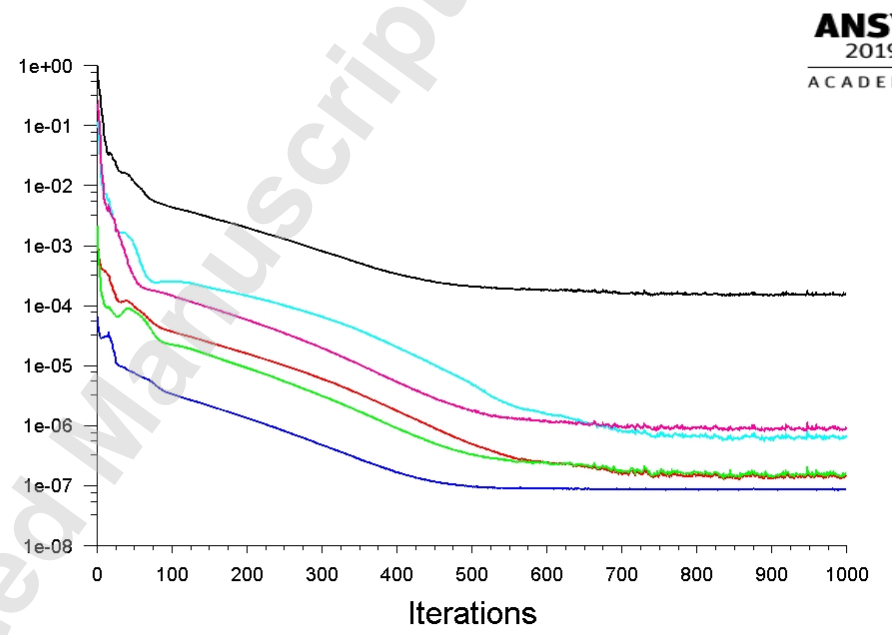

(b) Final deformed

Fig. 29. CFD residuals of the baseline blade and final deformed blade (Case 1). 


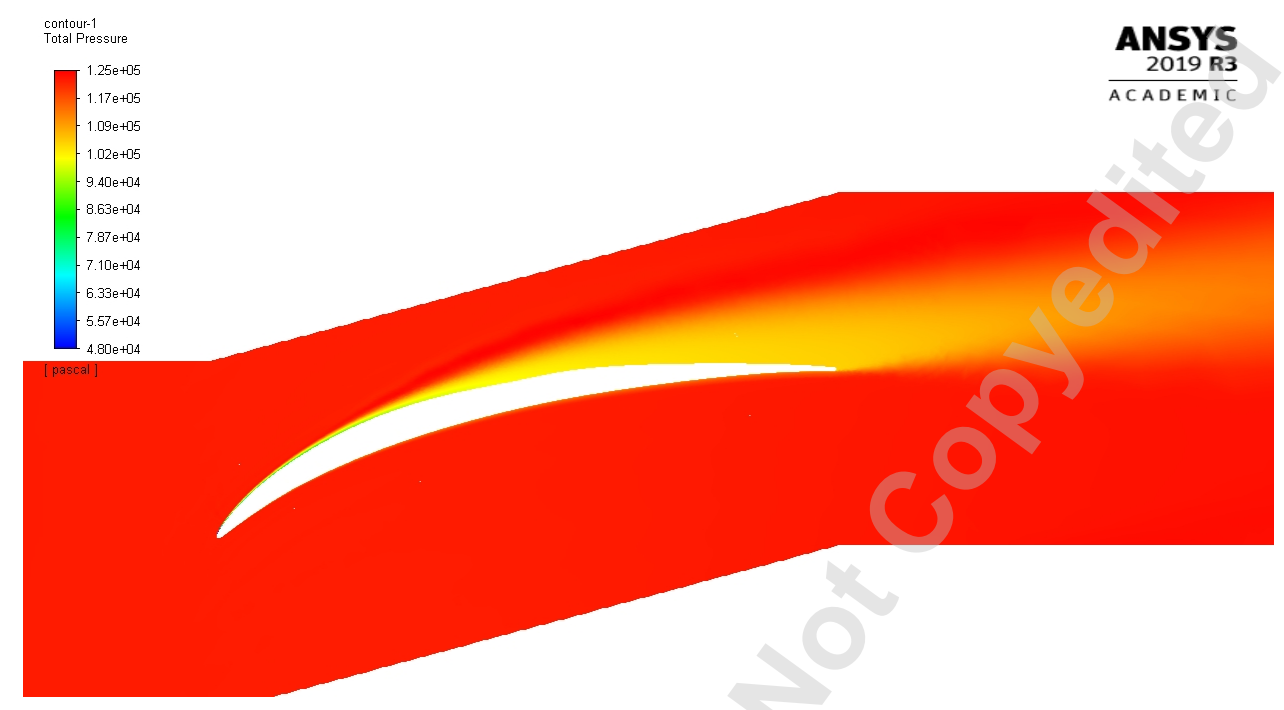

(a) Baseline blade

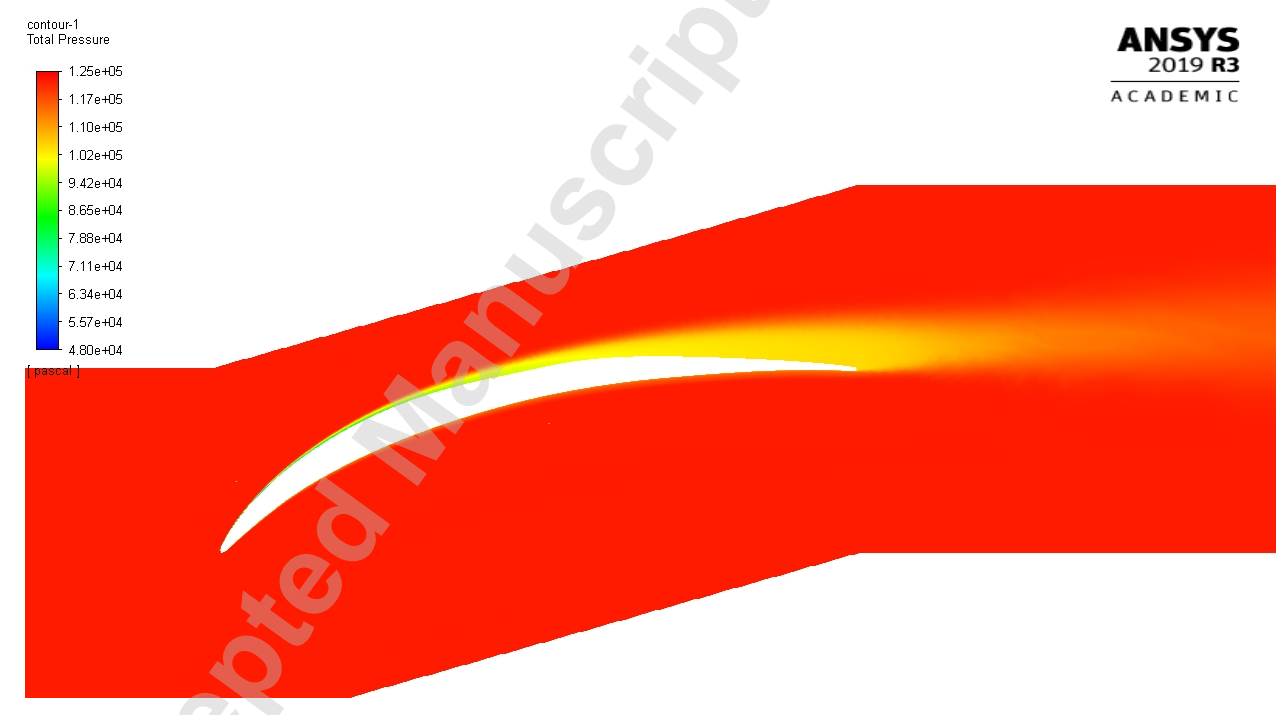

(b) Final deformed blade

Fig. 30. Total pressure distribution of the baseline blade and final deformed blade (Case 1). 


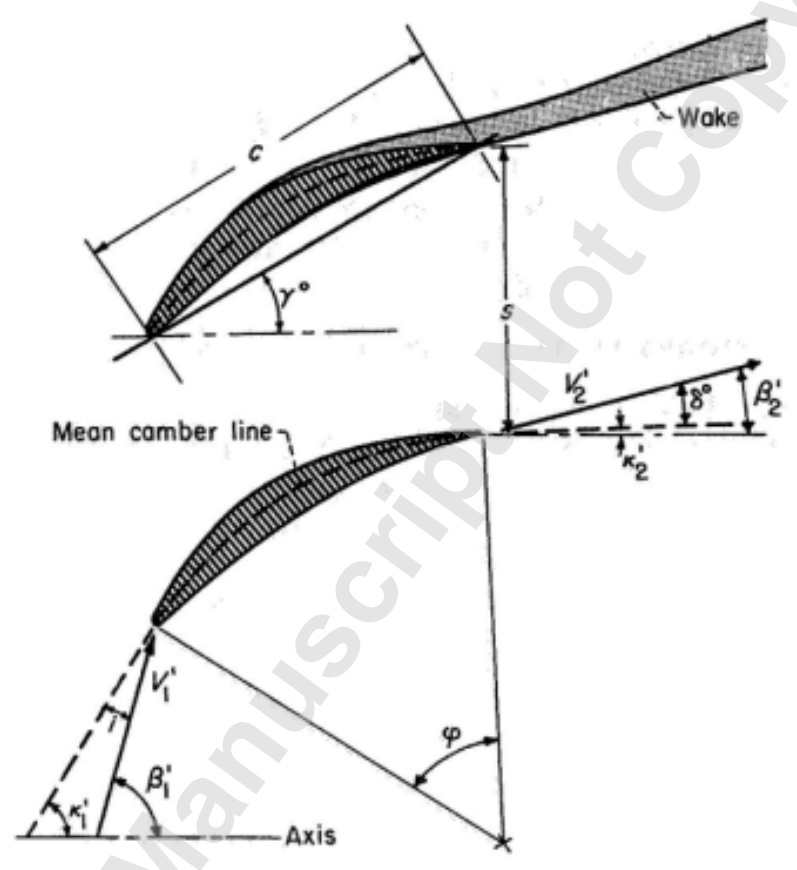

Fig. 31. Angles in a compressor cascade. 